\title{
Mobility-Based Clustering Algorithm for Multimedia Broadcasting over IEEE 802.11p-LTE-enabled VANET
}

\author{
Mohammad Syfullah ${ }^{1 *}$, Joanne Mun-Yee Lim $^{2}$ and Fei Lu Siaw ${ }^{1}$ \\ ${ }^{1}$ Center of Communication \& Intelligent Systems (CCIS), Faculty of Engineering and Built Environment, \\ SEGi University, Kota Damansara, Petaling Jaya 47810 - Malaysia \\ [e-mail: syfullah@hotmail.co.uk] [e-mail: siawfeilu@segi.edu.my] \\ ${ }^{2}$ School of Engineering, Department of Electrical and Computer Systems Engineering, \\ Monash University Malaysia, Bandar Sunway 47500 - Malaysia \\ [e-mail:Joanne.Lim@monash.edu] \\ *Corresponding author: Mohammad Syfullah
}

Received April 9, 2018; revised September 5, 2018; accepted October 11, 2018; published March 312019

\begin{abstract}
Vehicular Ad-hoc Network (VANET) facilities envision future Intelligent Transporting Systems (ITSs) by providing inter-vehicle communication for metrics such as road surveillance, traffic information, and road condition. In recent years, vehicle manufacturers, researchers and academicians have devoted significant attention to vehicular communication technology because of its highly dynamic connectivity and self-organized, decentralized networking characteristics. However, due to VANET's high mobility, dynamic network topology and low communication coverage, dissemination of large data packets (e.g. multimedia content) is challenging. Clustering enhances network performance by maintaining communication link stability, sharing network resources and efficiently using bandwidth among nodes. This paper proposes a mobility-based, multi-hop clustering algorithm, (MBCA) for multimedia content broadcasting over an IEEE 802.11p-LTE-enabled hybrid VANET architecture. The OMNeT++ network simulator and a SUMO traffic generator are used to simulate a network scenario. The simulation results indicate that the proposed clustering algorithm over a hybrid VANET architecture improves the overall network stability and performance, resulting in an overall 20\% increased cluster head duration, $20 \%$ increased cluster member duration, lower cluster overhead, $15 \%$ improved data packet delivery ratio and lower network delay from the referenced schemes [46], [47] and [50] during multimedia content dissemination over VANET.
\end{abstract}

Keywords: Vehicular ad-hoc network (VANET), LTE, clustering, Intelligent Transportation System (ITS), hybrid architecture. 


\section{Introduction}

Vehicle Ad-hoc Network (VANET) is the core element behind the latest developments in vehicular communication technology [1]. It is one of the fundamental components of the Intelligent Transportation System (ITS) technology [2]. It is a distinct category of Mobile Ad-hoc Network (MANET) [4], which consists of a set of high-speed and high-mobility vehicles equipped with sensors and communication devices [5]. It transforms each neighboring vehicle into a wide-range, wireless, ad-hoc network that enables vehicles to establish interconnection links and share information with each other wirelessly using a dedicated short-range communication (DSRC) protocol [6-8]. VANET can significantly enhance the driving experience by supporting critical vehicular safety applications, such as emergency or hazard warning, vehicle collision avoidance, traffic status broadcast, road condition sharing and lane-changing assistance [3].

Most of the applications in VANET require a real-time, up-to-date response from nearby vehicles [12]. Since VANET has distinctive characteristics, such as dynamic topology, frequent network disconnection, constrained mobility [9, 10] and limited capacity to disseminate road safety information, it has difficulty disseminating larger data packets (e.g. multimedia content) over the network. The major challenges include (1) mobility management, (2) broadcast storm problems [13], (3) network disconnection problems [10, 11], (4) network coverage, (5) data dissemination techniques and (6) high bandwidth. To keep a moving vehicle's connectivity high, many researchers have suggested clustering methods [21-54] that solve broadcast storm issues over VANET. To address network instability issues, store-carry-forward methods $[13,14]$ are commonly used in VANET.

Clustering in VANET is defined as a group of vehicles or nodes moving in the same direction that maintain communication links with each other without compromising network connection [9]. Clustering enhances a network's communication efficiency by making network management more convenient for VANET applications, along with vehicles' resource-sharing capabilities [15]. Re-clustering causes data loss and network overhead issues in a vehicular network environment, which makes the network unstable.

The utilization of cellular communications technology in vehicular networks has recently been investigated, which signifies as a contrasting option to the current IEEE 802.11p standard VANET. The IEEE 802.11p-based VANET, with high data rates and large coverage of cellular communication technology, has motivated researchers to examine the combination of both technologies [16, 17].

This paper proposes a mobility-based clustering algorithm to support efficient multimedia content dissemination with a reduced number of cluster heads (CHs) over a hybrid VANET architecture. To improve data dissemination efficiency, network delays and data packet delivery ratios (DPDR) over VANET, the proposed architecture presents a combination of traditional IEEE 802.11p standard and fourth-generation cellular technology Long-Term Evolution (LTE) to form a hybrid VANET.

We proposes a clustering algorithm for disseminating multimedia content over a hybrid VANET architecture, constructed using V2V and V2I message dissemination techniques in an urban traffic environment. The paper's contributions are listed as follows: 
1. A mobility-based clustering algorithm is proposed for multimedia content dissimination over an IEEE 802.11p-LTE-enabled VANET architecture. Mobility-based clustering is primarily influenced by direction, number of neighbors, distance and vehicle leadership.

2. Direct communication with CHs and secondary cluster heads (SE_CHs) is proposed to increase the network lifetime of nodes.

3. A cluster handshake process is introduced to overcome the affiliation issue in nodes over the vehicular network.

4. The proposed clustering algorithm is simulated in a realistic mobility scenario, which is generated with a vehicular traffic generator SUMO [18] and implemented in a network simulator OMNeT++ [19], along with a vehicular network framework Veins [20].

5. Extensive analysis is performed using the proposed clustering hybrid VANET architecture. In the analysis section, the proposed clustering algorithm and existing algorithms are compared using performance metrics, such as $\mathrm{CH}$ duration, cluster member (CM) duration, cluster overhead, DPDR and network delay.

The rest of this paper is organized as follows. Related studies are discussed in Section 2. A network scenario and a system model of the proposed hybrid VANET architecture are described in Section 3. The proposed stable, multi-hop clustering scheme is presented in Section 4. Performance analysis and simulation results comparing the proposed hybrid architectures are shown in Section 5. Finally, concluding remarks and future work are discussed in Section 6.

\section{Related Work}

Clustering algorithms were initially proposed in the late 1980s to organize interconnected nodes in MANET [21]. Until now, a wide range of clustering algorithms has been proposed, including mobility-based, network topology-oriented, code division-based, weight-based, direction-based, location-based and neighborhood-based [22-29]. However, these algorithms differ significantly from VANET clustering. Table 1 presents a summary of popular clustering algorithms proposed by fellow researchers. A vehicular clustering algorithm is performed by associating the vehicles moving into small groups, where coordinated communication uses a predefined, set rules of the cluster leader or CHs [15]. In each cluster, vehicles define only one $\mathrm{CH}$ and a few $\mathrm{CM}$ are selected. The $\mathrm{CH}$ functions as an access point for CMs to facilitate data dissemination among vehicles as well as among road-side units (RSUs).

A notable clustering algorithm was introduced by the Defense Advanced Research Projects Agency (DARPA) packet-radio network [30], whose goal was to solve MANET's fundamental problems by forming subnets autonomously within the network. Later, the lowest ID and highest degree (LID/HID) clustering algorithm was proposed by Gerla et al. [31], where each of the participating nodes contains a unique ID and a node has the lowest ID, stated as the $\mathrm{CH}$ [25]. Basu et al. [23] introduced another popular clustering algorithm mobility-based clustering algorithm (MOBIC). The authors developed this algorithm based on the previously proposed lowest-ID algorithm, in which increased successive data reception, a signal power-level mobility metric, has been used. MOBIC is mostly designed for MANET because of its simple design and because it does not scale well in the VANET environment. Similarly, several clustering algorithms proposed later, including distributed group mobility adaptive (DGMA) clustering [32] and MobHiD [33], each of which is designed for a MANET environment, present a progressive development in network performance under simple mobility models. 
As VANET research gradually moved away from its MANET background, clustering formation approaches diversified. Shea et al. proposed a unique clustering approach, named affinity propagation in vehicular environments (APROVE) [34], which applied a statistical analysis technique, affinity propagation (AP), for clustering. In this algorithm, the clustering establishment depends on proximity and the relative velocity of neighbouring vehicles. However, since APROVE is not a synchronous algorithm, the messages are not received in real time, which causes repeated transmissions.

Direction-based clustering algorithms for vehicular networks were presented in [35], where the authors proposed that each vehicle uses an embedded map to estimate the path direction as per their origin address and final stop. While vehicles going to the same destination form a cluster, this clustering approach only considers the direction of neighbouring vehicles to form a cluster, which causes rapid cluster changes. Subsequently, Goonewardene et al. proposed a robust mobility adaptive clustering scheme [36], where lower relative speeds, smaller inter-vehicle distances, and higher link-termination times considered performing clustering. This results in larger clusters with longer average affiliations.

Similarly, the authors in $[37,38]$ proposed a clustering scheme particularly designed for highway vehicle mobility, called threshold-based clustering. In their algorithm, the slowest vehicle from the neighbouring vehicles has to initiate the clustering process. The $\mathrm{CH}$ selection technique is based on HD [31], where proximity to the mean position value and the velocity of a vehicle are considered. This results in more members in each cluster.

The authors in [39] proposed a location-based aggregate local mobility (ALM) clustering scheme, which is a more developed version of the previously proposed MOBIC algorithm [23]. In ALM, the authors modified the MOBIC clustering process by implementing GPS location data into the system. Likewise, the authors in $[40,41]$ introduced a clustering algorithm for VANET dependent on GPS location data. In most places where location tracking services are either not available or might be available, service precision significantly varies. Imprecision in vehicle positioning, even of only a few meters, can severely affect cluster stability, leading to communication link breakdowns in the network.

Most of the abovementioned clustering schemes in the proposed vehicular environment are based on a one-hop clustering structure, where vehicles can communicate with only one-hop neighbouring vehicles. Subsequently, many clusters are formed with a small communication coverage, resulting in decreased cluster stability. Recently, a multi-hop clustering algorithm that improves network clustering performance has been investigated, by decreasing the cluster number and cluster overhead and enhancing cluster coverage.

Hafeez et al. [42] presented a new clustering scheme for VANET architecture, where vehicle speed becomes the key element in forming a cluster. In the algorithm, the authors tried to improve the performance of cluster stability using fuzzy logic-based $\mathrm{CH}$ selection. The algorithm was designed for low-mobility scenarios, which do not support rapid changes in network topology.

Zhang et al. [43] proposed a multi-hop clustering scheme based on vehicle mobility, where vehicles calculate the relative speed among neighbouring vehicles and the communication link, known as an algorithm metric. The algorithm generates cluster overhead during clustering control message dissemination over the network, which may affect network performance.

In [44], the authors introduced distributed multi-hop clustering based on neighborhood flow for VANET. In the algorithm, cluster formation depends on a neighbourhood concept, called 'neighbourhood follow', and clusters are larger than those in the previously proposed algorithms, which may negatively impact network stability. 
Table 1. Related Work on VANET Clustering

\begin{tabular}{|c|c|c|c|c|c|c|}
\hline Algorithm Name / Acronym & $\begin{array}{l}\text { No. of } \\
\text { Hops }\end{array}$ & $\begin{array}{l}\text { Clustering } \\
\text { Metric } \\
\text { Based On: }\end{array}$ & $\begin{array}{l}\text { Cluster } \\
\text { Update }\end{array}$ & Mobility & Open Issues & Citation \\
\hline $\begin{array}{l}\text { Mobiity Based Metric for } \\
\text { Clustering in MANET }\end{array}$ & Two & Mobility & Periodic & Distributed & Designed for MANET & [23] \\
\hline $\begin{array}{l}\text { Adaptive Clustering for Mobile } \\
\text { Wireless Networks }\end{array}$ & One & $\begin{array}{l}\text { Code-divisi } \\
\text { on }\end{array}$ & - & Dynamic & Designed for MANET & [25] \\
\hline $\begin{array}{l}\text { Mobility Prediction-Based } \\
\text { Clustering (MPBC) } \\
\end{array}$ & One & Direction & Periodic & Dynamic & Designed for MANET & [26] \\
\hline $\begin{array}{c}\text { Lowest ID/Highest Degree } \\
\text { (LID/HID) }\end{array}$ & One & Mobility & Periodic & Dynamic & Designed for MANET & {$[31]$} \\
\hline $\begin{array}{c}\text { Distributed Group Mobility } \\
\text { Adaptive Clustering (DGMA) }\end{array}$ & One & Direction & Periodic & Distributed & Designed for MANET & [32] \\
\hline $\begin{array}{l}\text { Neighborhood Stability-Based } \\
\text { Mobility Prediction (MobHiD) }\end{array}$ & One & Speed & Periodic & $\begin{array}{c}\text { Car } \\
\text { Following }\end{array}$ & Designed for MANET & [33] \\
\hline $\begin{array}{l}\text { Affinity Propagation in Vehicular } \\
\text { Environment (APROVE) }\end{array}$ & Multi & $\begin{array}{l}\text { Location, } \\
\text { Speed } \\
\text { Difference }\end{array}$ & - & Dynamic & $\begin{array}{l}\text { Synchronizing, Repeated } \\
\text { Transmission }\end{array}$ & [34] \\
\hline Density Based Clustering (DBC) & Two & Location & Periodic & Dynamic & $\begin{array}{c}\text { Increased cluster overhead, } \\
\text { load balancing }\end{array}$ & [35] \\
\hline Direction Based Clustering & One & Direction & Periodic & $\begin{array}{c}\text { Car } \\
\text { Following }\end{array}$ & $\begin{array}{l}\text { Rapid cluster change over, } \\
\text { link stability }\end{array}$ & {$[36]$} \\
\hline $\begin{array}{l}\text { Robust Mobility Adaptive } \\
\text { Clustering (RMAC) }\end{array}$ & One & Location & Periodic & Dynamic & $\begin{array}{l}\text { Large cluster size, long } \\
\text { affiliation time }\end{array}$ & [37] \\
\hline $\begin{array}{l}\text { Threshold-Based Clustering } \\
\text { (TBC) }\end{array}$ & One & $\begin{array}{l}\text { Direction, } \\
\text { Speed } \\
\text { Difference }\end{array}$ & Periodic & $\begin{array}{c}\text { Car } \\
\text { Following }\end{array}$ & $\begin{array}{c}\text { High number of cluster } \\
\text { member, network overhead }\end{array}$ & {$[38]$} \\
\hline Aggregate Local Mobility (ALM) & Multi & Location & Periodic & Dynamic & $\begin{array}{c}\text { Location accuracy, } \\
\text { unavailability of GPS }\end{array}$ & [39] \\
\hline $\begin{array}{l}\text { Multi homing Clustering } \\
\text { (MHCV) }\end{array}$ & Multi & Direction & - & $\begin{array}{c}\text { Car } \\
\text { Following }\end{array}$ & $\begin{array}{c}\text { Location accuracy, } \\
\text { unavailability of GPS data }\end{array}$ & [40] \\
\hline Modified DMAC Clustering & Multi & $\begin{array}{c}\text { Speed } \\
\text { Difference }\end{array}$ & Periodic & $\begin{array}{c}\text { Car } \\
\text { Following }\end{array}$ & $\begin{array}{l}\text { Location accuracy, } \\
\text { unavailability of GPS }\end{array}$ & [41] \\
\hline $\begin{array}{l}\text { Fuzzy Logic-Based Cluster head } \\
\text { selection algorithm }\end{array}$ & Two & Direction & Periodic & Dynamic & $\begin{array}{l}\text { High mobility, network } \\
\text { topology }\end{array}$ & [42] \\
\hline $\begin{array}{l}\text { Vehicle Mobility-Based } \\
\text { Clustering Algorithm } \\
\end{array}$ & Multi & Direction & Periodic & Dynamic & High overhead & [43] \\
\hline $\begin{array}{c}\text { Distributed Multi-Hop Clustering } \\
\text { Based on Neighborhood Follow } \\
\text { (DMCNF) }\end{array}$ & Multi & Location & Periodic & $\begin{array}{c}\text { Car } \\
\text { Following }\end{array}$ & $\begin{array}{l}\text { Network stability, high } \\
\text { overhead, large cluster }\end{array}$ & [44] \\
\hline $\begin{array}{l}\text { Vehicular Multi-Hop Algorithm } \\
\text { for Stable Clustering (VMaSC) }\end{array}$ & Multi & Direction & Periodic & $\begin{array}{c}\text { Car } \\
\text { Following } \\
\end{array}$ & $\begin{array}{l}\text { High overhead, Faulty } \\
\text { affiliation }\end{array}$ & [45] \\
\hline $\begin{array}{c}\text { Vehicular Multi-Hop Algorithm } \\
\text { for Stable Clustering on Hybrid } \\
\text { Vehicular Network }\end{array}$ & Multi & Direction & Periodic & $\begin{array}{c}\text { Car } \\
\text { Following }\end{array}$ & Faulty affiliation & [46] \\
\hline $\begin{array}{l}\text { Distributed D-Hop Cluster } \\
\text { Formation (DHCV) }\end{array}$ & Multi & $\begin{array}{l}\text { Location, } \\
\text { Speed } \\
\text { Difference }\end{array}$ & Periodic & Dynamic & $\begin{array}{c}\text { Unorganized network, high } \\
\text { overhead }\end{array}$ & [47] \\
\hline $\begin{array}{c}\text { Distributed Cluster Formation } \\
\text { based on End-to-End Relative } \\
\text { Mobility (DHCVE) }\end{array}$ & Multi & Location & Periodic & Dynamic & $\begin{array}{c}\text { Random topology change, } \\
\text { network storm }\end{array}$ & [48] \\
\hline $\begin{array}{c}\text { Decentralized Clustering using } \\
\text { Adaptive Resonance Theory } \\
\text { (ART) }\end{array}$ & Multi & Direction & Periodic & $\begin{array}{c}\text { Car } \\
\text { Following }\end{array}$ & $\begin{array}{l}\text { Random topology change, } \\
\text { network storm }\end{array}$ & [50] \\
\hline $\begin{array}{l}\text { Agent based Dynamic Clustering } \\
\text { for Hybrid VANET (ADCHV) }\end{array}$ & Multi & Dynamic & Periodic & Dynamic & $\begin{array}{l}\text { High cost, routing } \\
\text { efficiency }\end{array}$ & [51] \\
\hline
\end{tabular}


Ucar et al. [45] initially proposed a vehicular multi-hop algorithm for stable clustering (VMaSC), which considers relative vehicle mobility as the main element for cluster formation. Later, the authors extended the algorithm in [46] to incorporate an LTE-enabled vehicular network. In this algorithm, bandwidth consumption of data dissemination in high density is extreme, which affects cluster reliability and data transmission speeds. Furthermore, the use of periodic Hello messages without handshaking for affiliation can cause isolated nodes to suffer from faulty affiliation.

In [47], the authors described a D-hop clustering algorithm for VANET (DHCV), where vehicles are arranged into adaptive-sized groups according to their respective mobility to perform non-overlapping clustering. The authors also proposed that vehicles choose their $\mathrm{CH}$ by calculating relative mobility within D-hop neighbourhoods. Subsequently, the authors developed another distributed clustering algorithm named DCEV in [48], where the cluster formation was similar to that in the DHCV algorithm. In the algorithm, the relative mobility was calculated based on end-to-end vehicle mobility in the same neighbourhood. Afterwards, the authors implemented a DCEV algorithm on a vehicular cloud model in [49]. However, the non-overlapping, clustering technique with adaptive cluster size results in a disorganized network with a high overhead.

In [50], the authors presented a decentralized clustering algorithm for VANET, based on adaptive resonance theory (ART), where vehicles create miniclusters that are in the same communication range as that of the other vehicles. Based on the authors' proposal, the miniclusters were created based on speed, where the vehicles were categorized as either high-, medium- or low-speed. ART is an unsupervised neural network model, which increases the network storm problem due to random changes in network topology.

Hubballi et al. [51] presented an agent-based clustering algorithm named agent-based dynamic clustering for hybrid VANET (ADCHV). The authors mentioned dynamic clustering as a method where vehicles create a cluster and move in a direction to keep the dynamic topology stable. In the algorithm, the vehicle agent forms a cluster and maintains $\mathrm{CH}$ changeover. This increases network maintenance expense and affects dissemination performance and routing efficiency.

Compared to one-hop and multi-hop clustering from the abovementioned algorithms, multi-hop clustering can enlarge the cluster communication range and achieve additional development in VANET environments. The most recently proposed clustering algorithms show major improvements in clustering techniques and processes. However, remaining open issues, which need to be resolved, can be isolated into five major categories: 1) To avoid network congestion, clustering algorithms should minimize repeated transmissions. 2) To strengthen network stability, clustering algorithms should reduce rapid cluster changeovers. 3) To improve network performance, clustering algorithms should lower the overhead during clustering formation and maintenance processes. 4) To reduce long affiliation time, the algorithm should use smaller cluster sizes. 5) The algorithms should be capable of providing higher data throughputs over VANET without compromising network performance.

\section{Network Scenario and System Model}

In developing a mobility-based, multi-hop clustering scheme, it is assumed that all vehicles in the network are equipped with on-board units (OBU) devices. The vehicles are also equipped with speed- and distance-measuring devices, such as iBeacon location tracking devices [52], GPS devices and digital odometers. Those devices are commonly available on vehicles to 
track movement and distance travelled. Vehicles and RSUs in the network are expected to be interconnected if the inter-vehicle distance is smaller than the vehicles' transmission ranges. Vehicles are also assumed to travel in straightforward, roundabout and orthogonal junctions. The streets are split into multiple traffic lanes, and vehicles traveling in adjoining paths move in the same direction, as shown in Fig. 1. Inter-vehicle distances, movement direction estimations and beacon scheduling processes are discussed in the following sections.

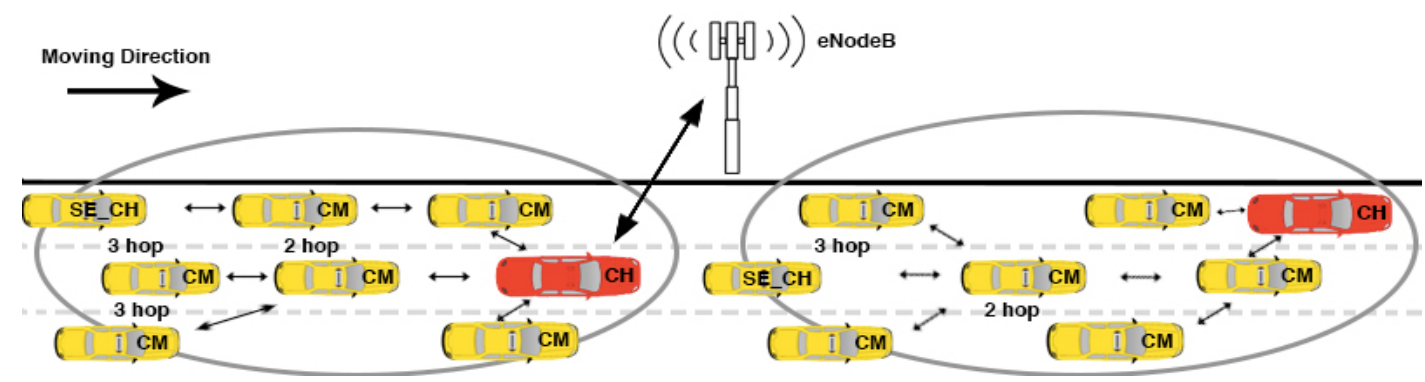

Fig. 1. Hybrid VANET Architecture Network Scenario

\subsection{Distance and Movement Direction Estimation}

In the network, each vehicle broadcasts beacon messages or HELLO_PACKETs periodically, which contain information such as vehicle identification, present status, road direction and time interval. In Fig. 2, a vehicle $W$ is located at a road position where beacon messages are transmitted from vehicles $t$ and $B$. These are received by vehicle $W$, with a time interval $\boldsymbol{t}$, which estimates their distance from $\angle$ and $B$, i.e. $d_{A t}$ and $d_{B}$, respectively, by using the ranging technique. Time intervals signify the time taken in-between packets generated from vehicles $\mathrm{A}$ and $\mathrm{B}$.

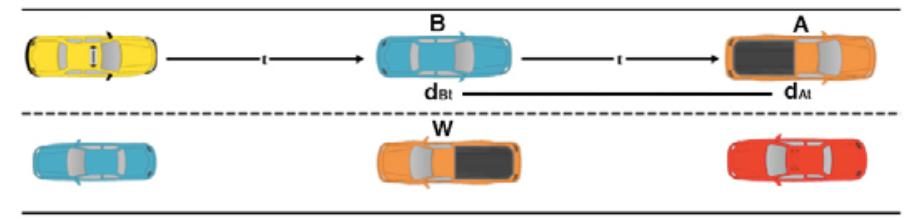

Fig. 2. Estimation of Vehicle Movement Direction

To estimate the inter-vehicle distance for two possible vehicles, corresponding interception points are obtained, i.e. $D_{A}\left(x_{A t}, y_{A t}\right)$ and $D_{B}\left(x_{B t}, y_{B t}\right)$. The equations representing the distance between the two points are expressed as

$$
\begin{aligned}
& d_{A t}=\sqrt{\left(x_{B}-x_{A}\right)^{2}+\left(y_{B}-y_{A}\right)^{2}} \\
& d_{B t}=\sqrt{\left(x_{A}-x_{B}\right)^{2}+\left(y_{A}-y_{B}\right)^{2}}
\end{aligned}
$$

However, given just one set of beacon messages, the receiving vehicle cannot be sure whether the vehicles are travelling in the same or opposite direction. Thus, to determine the vehicle's direction, $W$ waits until it receives the next set of broadcast messages from A and B, and then calculates the pairwise relative force for every vehicle applied to every axis $(x, y$, using the coulomb law.

$$
F_{r x}=k_{x} \times\left(\frac{q_{A} q_{B}}{r_{A}}\right)
$$




$$
F_{r y}=k_{y} \times\left(\frac{q_{A} q_{B}}{r_{B}}\right)
$$

where $k_{x}, k_{y}$ are coulomb's constants, $q_{A}$ and $q_{B}$ indicate parameters that show whether the force among the vehicles is positive or negative (depending on whether the vehicle is approaching or moving away) and $r_{A}, r_{B}$ represent the distance between the vehicles. On the basis of this new information, $W$ obtains two possible positions, which are used to estimate the direction of these vehicles.

\subsection{Beacon Scheduling}

Because of the huge number of beacon messages transmitted over the network from moving vehicles, to arrange vehicle mobility more efficiently, the network utilized a two-phase beacon schedule. The system uses two distinct time intervals for beacon message scheduling, where the message transmits for a short period and a stable period. The distinct time interval ' $t$ for both the short and stable periods considers ' $T$, where $T \geq t$.

\section{Proposed Clustering Algorithm MBCA}

The main goal of clustering is to minimize the number of communications over a vehicular network. This paper proposes a scalable clustering algorithm for multimedia content dissemination over a hybrid VANET architecture. The proposed clustering algorithm eventually helps in providing stable clustering by reducing network overhead, avoiding inter-cluster congestion, reducing repeated transmissions, increasing cluster node lifetime, reducing rapid cluster changeovers, ensuring network reliability and enabling higher data throughputs over VANET.

To reduce network overhead, the proposed clustering algorithm presents peer-to-peer communication with neighbouring vehicles, regardless of whether the vehicle is a $\mathrm{CH}$ or a member of an existing cluster. This also avoids sending multiple hello messages when joining the network. The algorithm maintains a stable cluster formation by calculating traffic flow, average relative speed, vehicle headings and CM size. This algorithm also reduces inter-cluster snooping by introducing cluster merging methods and minimizing cluster overlap on the network. The presence of SE_CHs in each cluster increases cluster node lifetime and reduces rapid cluster changeover. Our approach in constructing these clusters involves the following steps.

\subsection{Vehicle Status}

Every vehicle sends a beacon message periodically to its neighbouring vehicle to form and exchange their initial clustering information. Message dissemination for a vehicle depends on the status of its information over the network. Each vehicle in the network operates the following statuses at all times.

- PRIMARY STATE (PS), the starting status of a vehicle, which does not belong to any cluster. In this stage, vehicles broadcast HELLO_PACKETs to nearby vehicles.

- STATUS DETERMINATION $(S D)$ is where the vehicle information status makes a decision about the next stage.

- $\mathrm{CH}$ is the status where the vehicle is declared to be a cluster leader. Each cluster has only one $\mathrm{CH}$, which communicates with all CMs.

- SE_CH is the status where the last member of the cluster hop acts as a $\mathrm{CH}$ in case of $\mathrm{CH}$ failure or an out-of-coverage situation. 
- $C M$ is the status of a vehicle connected to an existing cluster. CM maintains communication with $\mathrm{CH}$ and is responsible for inter-cluster communication.

- SELF-CLUSTER HEAD (SELF_CH) is the status that acts as a cluster leader on a temporary basis. It is declared as $S E L F \_C H$ only when it is unable to join any cluster due to the maximum $\mathrm{CM}$, which is already reached in a cluster.

Table 2. List of Notations

\begin{tabular}{|c|c|}
\hline Symbol & Definition \\
\hline PS & Initial Status \\
\hline$S D$ & Status Determination \\
\hline $\mathrm{CH}$ & Cluster Head \\
\hline SE_CH & Secondary Cluster Head \\
\hline$C \bar{M}$ & Cluster Member \\
\hline VInfo & Vehicular Information \\
\hline AVEREL_SPEED & Average Relative Speed of Vehicle \\
\hline RELATIVE_FORCE & Relative Force of Vehicle \\
\hline TRY_CONNEECT & Try Connect Flag for Vehicle \\
\hline JOIN_REQ & Join Request Packet \\
\hline$J O I N \_R E S P$ & Join Response Packet \\
\hline RELATIVE_VELOCIRY & Relative Velocity of Vehicle \\
\hline MAX_HOP & Maximum Hop between $\mathrm{CH}$ and $\mathrm{CM}$ \\
\hline$M A X \_N U M B E R$ & Maximum Number of Cluster Member \\
\hline HELLO_PACKET & Periodic Hello Packet \\
\hline$L E A D E \bar{R}$ & Cluster Leader \\
\hline$M E M B E R$ & Cluster Member \\
\hline$V_{\text {status }}$ & Vehicle Status \\
\hline SELF_CH & Self-Declared CH \\
\hline JOIN_REQ_FORWARD & Forward Join Request Packet \\
\hline$M E R G E \_R E Q$ & CH’s Merge Request \\
\hline COMBINE_TIMER & Cluster State Timer \\
\hline CLUSTER_DETAILS & Cluster Information \\
\hline COMBINE_REQ & Cluster Merge Request \\
\hline TIME_INTERVAL & State Time Interval \\
\hline
\end{tabular}

\subsection{Vehicle Information and Neighboring Sensing}

Vehicle information (VInfo) refers to information related to each node, and mostly contains the vehicle's identity, position, direction, velocity, current clustering status, cluster ID, number of hops in $\mathrm{CH}$ (if the vehicle is a $\mathrm{CM}$ ) and its clustering metric. VInfo is updated according to vehicular status changes or HELLO_PACKET responses from neighbouring vehicles within MAX_HOP, as shown in Algorithm 1. MAX_HOP represents the maximum number of hops between $\mathrm{CH}$ and $\mathrm{CM}$ in the same cluster.

Neighbourhood identification is the process by which a vehicle or node recognizes its current neighbours within its transmission range. Since all vehicles are moving, neighbour vehicle information is always changing. Neighbour information $n_{i}$ of vehicle $i$ is dynamic and updates its vehicular status very frequently. To detect its neighbours, each vehicle estimates its inter-vehicle distance, movement direction and average speed by broadcasting a hello packet or a beacon message periodically, which contains VInfo. Vehicles moving in the same direction are grouped in the same cluster, while those moving in opposite directions are not part of the cluster. The average relative speed for vehicle $i$ is calculated as

$$
\text { AVEREL_SPEED } D_{i}=\frac{\sum_{j=1}^{n_{i}}\left|s_{i}-s_{j}\right|}{n_{i}}
$$

where $n_{i}$ is the number of vehicles in the same lane within MAX_HOP. Vehicle $i$ identifies the $j$ th nearby vehicle, and $s$ is vehicle $i$ 's speed. 


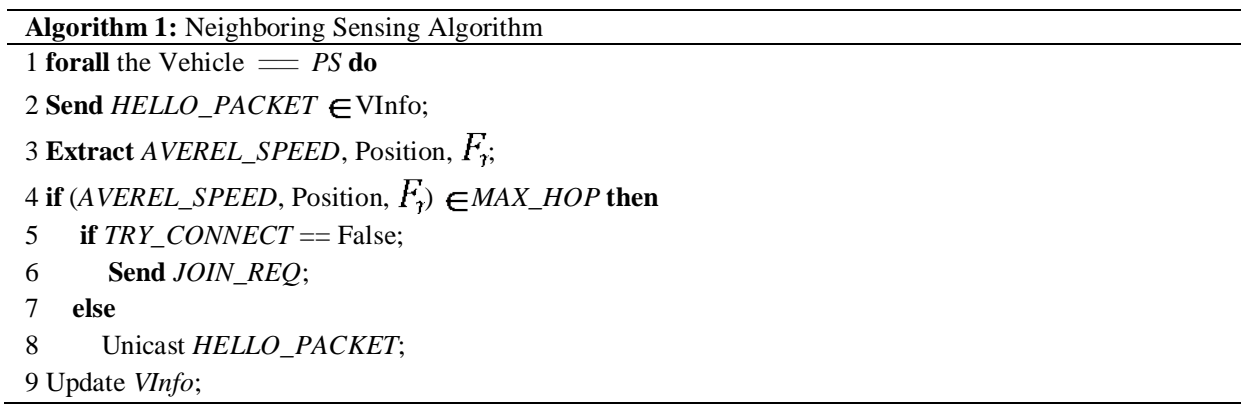

\subsection{Cluster Leadership}

Initially, each vehicle starts in the Primary State (PS) status. Periodic exchanges of hello packets help discover which vehicles own VInfo, along with its neighbours, and the change status to SD. According to the current status of the vehicle and neighbouring vehicles, each vehicle makes a decision about cluster formation, cluster maintenance and cluster leadership, as presented in Algorithm 2. A platooning controller formula is used to determine the cluster leader. This platooning method is dependent on the spacing among the vehicles, acceleration and the average speed of the vehicle leader.

According to Newton's law [53], the dynamic equation for the $i$-th subsequent vehicle in the platoon can be expressed as follows:

$$
m_{i} P_{i}=F_{i}+F_{i, g}+F_{\text {iaero }}+F_{i, d r a q}
$$

where $P_{1}$ represents the position of the $i$-th vehicle on the $\mathrm{X}$ axis, $m_{i}$ is the mass for the $i$-th vehicle, $F_{i}$ is the force produced by the $i$-th vehicle, $F_{i, q_{1}}$ is the acceleration of gravity of the $i$-th vehicle and $F_{\text {iner },}$ and $F_{i, d r a, q}$ represent aerodynamic and mechanical drag forces, respectively.

We denote inter-vehicle spacing of the $i$-th subsequent vehicle with regard to the $\mathrm{CH}$ vehicle as follows:

$$
\delta_{i}=\Delta X_{i}-l-\left(v_{i}-V\right)
$$

where $\delta_{i}$ is the inter vehicle spacing, $\Delta X_{i}=x_{i-1}-x_{i}$ is the real spacing between the $i$-th vehicle, $x_{1}$ is the position, $i$ is the desired inter-vehicle distance, $v_{1}$ is the velocity and $V$ is the velocity value shared between all vehicles at the same time.

To signify the aggregate relative velocity of the $i$-th vehicle, the equation is derived as follows:

$$
\delta_{i}=P_{0}-P_{i}-i \delta_{d}-i L
$$

where $\delta_{a}$ is the aggregate RELATIVE_VELOCITY for the vehicles, $\delta_{d}$ is the preferred vehicle spacing, $\mathrm{L}$ is the length of the vehicle and $P_{0}$ is the position of the vehicle $P_{r}$. The dynamic pattern of vehicle $i=1,2,3 \ldots \ldots . . . N-1$.

According to the vehicles' spatial position, a platoon can be classified into three roles, as shown below.

- Cluster Leader: The leader is the vehicle that leads the platoon on the road. It maintains the platoon's creation and controls the membership of other vehicles when they join or resign from the platoon.

- Members: These are the vehicles that receive information from the leader and relay it to other vehicles in the platoon.

- Secondary Leader: This vehicle is usually located at the end of the platoon. It is responsible for communicating with nearby vehicles and performing as a secondary 
platoon leader (when the platoon leader is not present), to convince others to join or merge with the platoon.

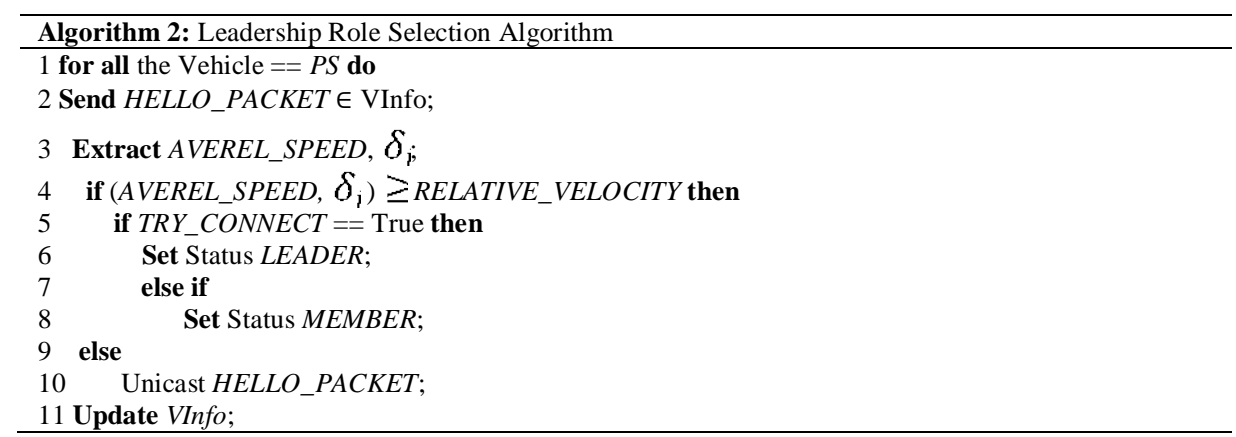

\subsection{Handshake with Clusters}

The handshake process with clusters starts right after a vehicle selects its $\mathrm{CH}$ in a hop. In multi-hop clustering, each $\mathrm{CH}$ keeps a complete list of CMs within its hop, where a CM only follows its $\mathrm{CH}$. In our proposed approach, a vehicle can identify a potential communication discrepancy with the $\mathrm{CH}$ or $\mathrm{CM}$ during the handshake process to ensure a communication link between the constructed clusters. The handshake process with clusters is explained in Fig. 3 [54], where a vehicle or node $N_{6}$ selects its $\mathrm{CH} N_{h}$ with an intermediate node $N_{i}$. In the handshake process, node $N_{\ell}$ sends a request to $N_{r_{i}}$ and waits for a response from the $\mathrm{CH}$. In the intermediate stage, the node $N_{i}$ receives a request message from node $N_{6}$ and forwards it to its $\mathrm{CH} N_{r}$ only if node $\mathrm{Ni}$ is not stated as the $\mathrm{CH}$ within the hop. The $\mathrm{CH} N_{r}$ determines whether it accepts the request. If so, it replies to $N_{0}$ to accept the request, and if not, $N_{r_{i}}$ discards the request. Thereafter, node $N_{i}$ forwards the reply to $N_{6}$. If node $N_{6}$ receives a reply from $N_{k}$ within a predefined time interval, the handshake is considered successful.

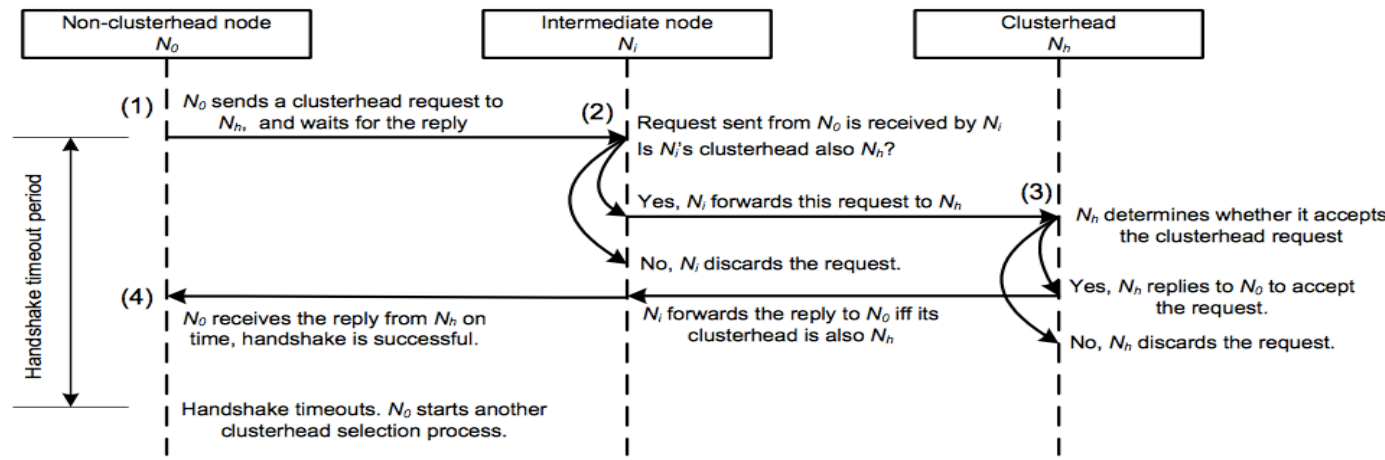

Fig. 3. Handshake Process with CHs [54]

\subsection{Cluster Status Changeovers}

As presented in Algorithm 3, a vehicle switches its status from SD to CM only if the vehicle receives a JOIN_RESP message from any of the secondary clusters, CMs or CHs. The representation of JOIN_RESP is considered a successful join request to a cluster. In each cluster, the last CM elected as an SE_CH becomes a CM when the $\mathrm{CH}$ loses the connection. Vehicles inside the cluster switch their status from $\mathrm{CM}$ to $\mathrm{SE} \_\mathrm{CH}$ when $\mathrm{CH}$ declares the last $\mathrm{CM}$ to any $\mathrm{CM}$. In Algorithm 3, a vehicle switches its status from SD to 
JOIN_REQ_FORWARD when it is unable to join any SE_CH, CH or CM, when the maximum number of CMs has been reached or when the vehicle is not traveling at an average speed similar to that of nearby vehicles.

The vehicle status SD switches to SELF_CH status if the JOIN_REQ_FORWARD condition is met. This SELF_CH status is different from the CHs, since a lake of CM connects with SELF_CH to become CH. A vehicle switches status from SELF_CH to CH if it receives JOIN_REQ from CM. The vehicle switches status from $\mathrm{CH}$ to $\mathrm{CM}$ when another neighbouring $\mathrm{CH}$ sends and accepts MERGE_REQ. The vehicle shifts from CM to SD status if the connection is lost.

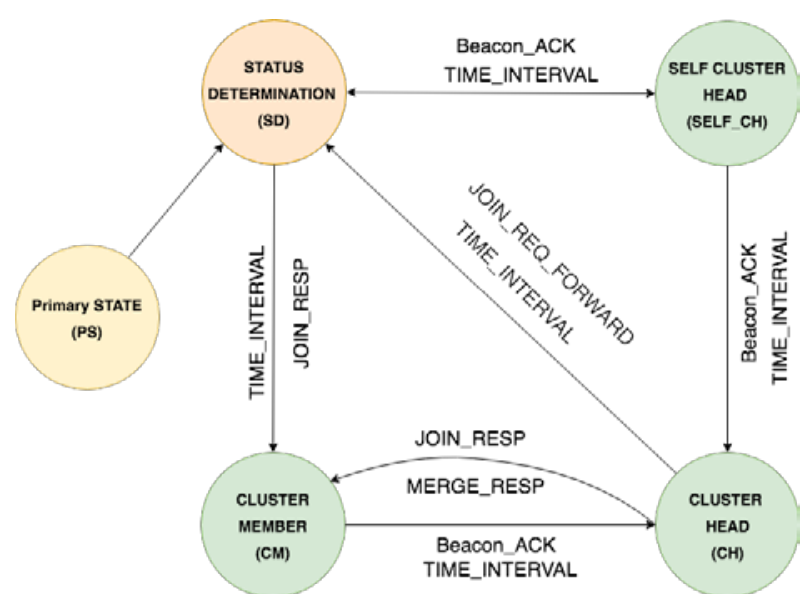

Fig. 4. Cluster Status Changeover

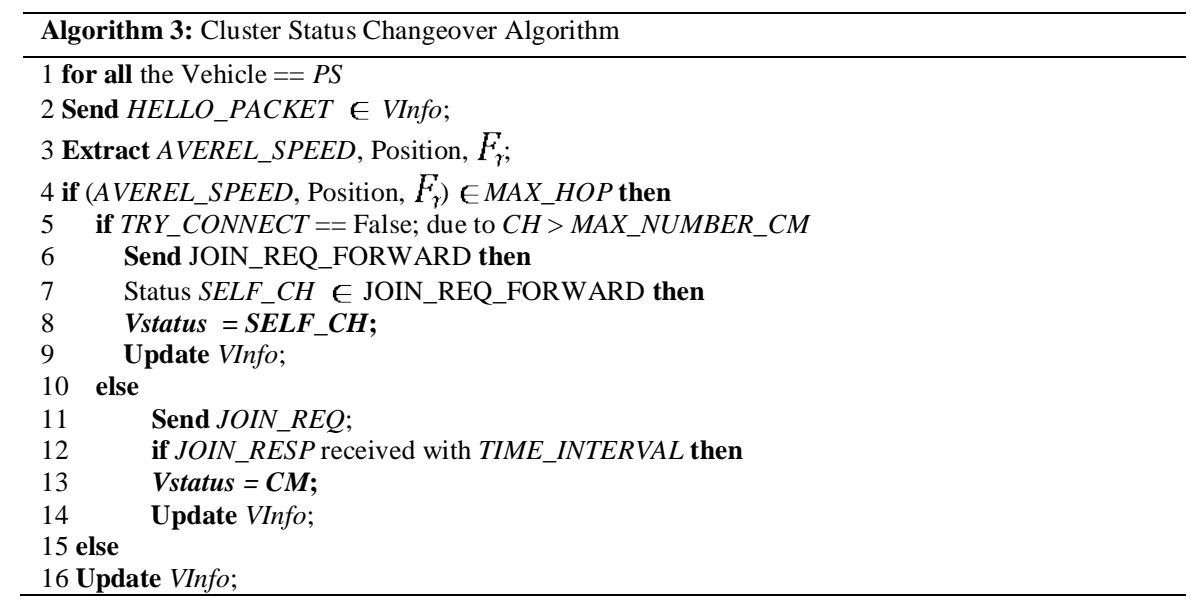

\subsection{Cluster Formation Process}

Similarly, as presented in Algorithm 4, to execute a cluster formation, each vehicle with SD status first tries to discover neighbouring vehicles by sending periodic HELLO_PACKET. This enables a vehicle to find an existing cluster and its CHs or CMs, which reduces the number of cluster leaders and network overload. If the existing $\mathrm{CHs}$ have fewer than the maximum number of members in a cluster, a vehicle can send JOIN_REQ. In normal time, CHs keep updating members about how many members are connected in the cluster and their capacity. This allows neighbouring vehicles to join a cluster more rapidly. 


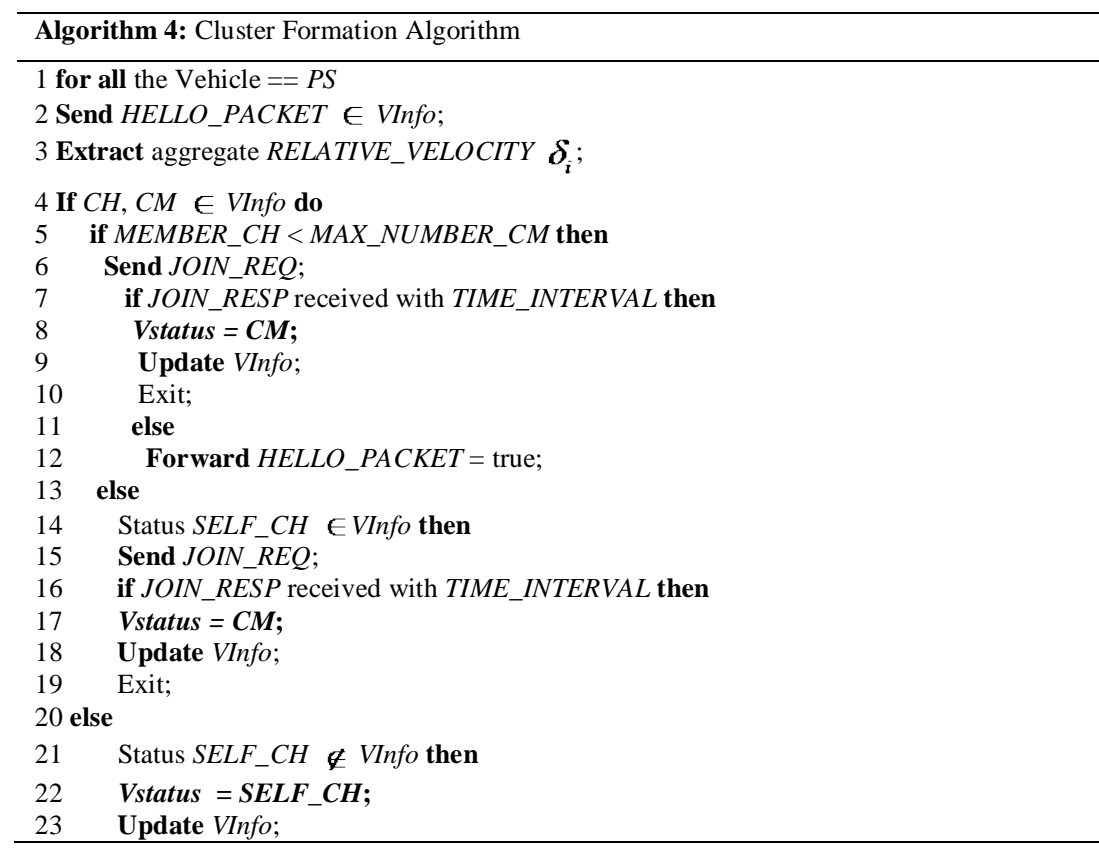

The $\mathrm{CH}$ determines whether it can accept the request. If CH replies with JOIN_RESP within a set time interval, then the vehicle changes its status to $\mathrm{CM}$. Otherwise, the vehicle unicasts JOIN_REQ to neighbouring clusters and updates VInfo. In the cluster, when the cluster leader loses communication with the $\mathrm{CMs}$, the $\mathrm{SE}$ _CH acts as a temporary $\mathrm{CH}$ and immediately selects a new $\mathrm{CH}$ from the existing $\mathrm{CM}$. If the vehicle is unable to join any existing cluster, it checks the neighbouring vehicles with SELF_CH status. If SELF_CH status is found, the vehicle tries to connect by sending JOIN_REQ. After getting JOIN_RESP from SELF_CH, it changes the status to CM. If no SELF_CH vehicles are found, the vehicle sets its status to SELF_CH.

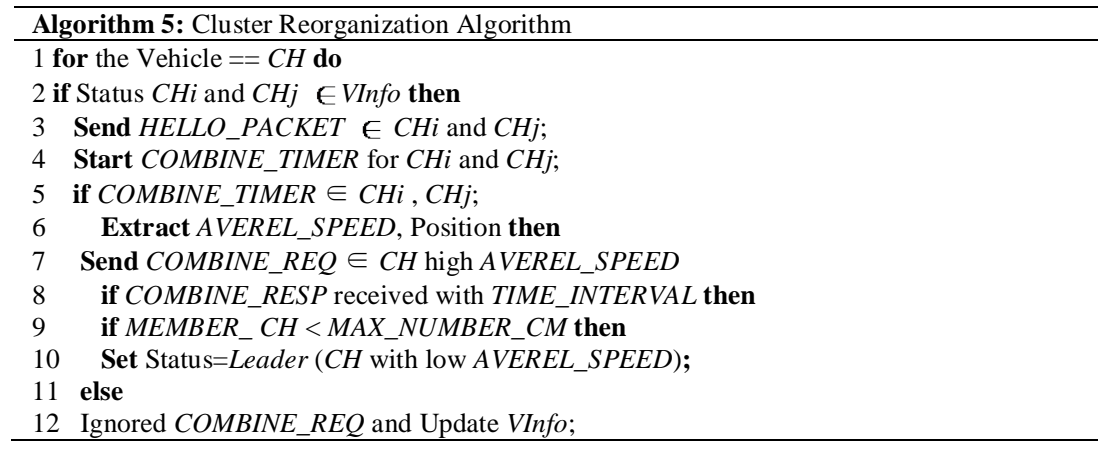

\subsection{Cluster Reorganization}

As presented in Algorithm 5, when a vehicle performing as a $\mathrm{CH}$ detects another $\mathrm{CH}$ in its range, it checks its neighbourhood duration by measuring COMBINE_ TIMER. If both CHs meet the COMBINE_TIMER duration, they share their CLUSTER_ DETAILS along with their average speeds. Both CHs check the possibility of combining clusters. To merge clusters, both must be moving in the same direction and the number of CMs must be less than the 
maximum. The $\mathrm{CH}$ with a higher average speed sends COMBINE_REQ and waits for a response from $\mathrm{CH}$ with a lower average speed, until TIME_INTERVAL duration has lapsed. If the request is accepted by both $\mathrm{CHs}$, the lower-average-speed $\mathrm{CH}$ becomes the leader of the newly combined cluster. If the COMBINE_REQ request is not accepted, it is ignored and $\mathrm{CH}$ continues.

\section{Performance Evaluation}

\subsection{Simulation Environment}

In this section, we validate the analytical results of our proposed system in the IEEE 802.11p-LTE standard hybrid VANET architecture. To produce a realistic vehicle mobility pattern, the open-source traffic simulator Simulation of Urban Mobility (SUMO) [18] and network simulator OMNeT++ [19] were used to simulate this proposed scheme. To support an IEEE 802.11p standard network in a vehicular network simulation, a Veins framework [20] and an INET [20] framework were implemented. This enables IEEE 802.11p-LTE standard networks in the simulation.

A SUMO [18] traffic simulator, developed by Garman Aerospace Center, is capable of simulating an individual driver's behaviour on the road. Veins [20] is an open-source simulation framework of inter-vehicular communication that includes a network simulator OMNeT++ [19] and a realistic road traffic simulation model SUMO [18].

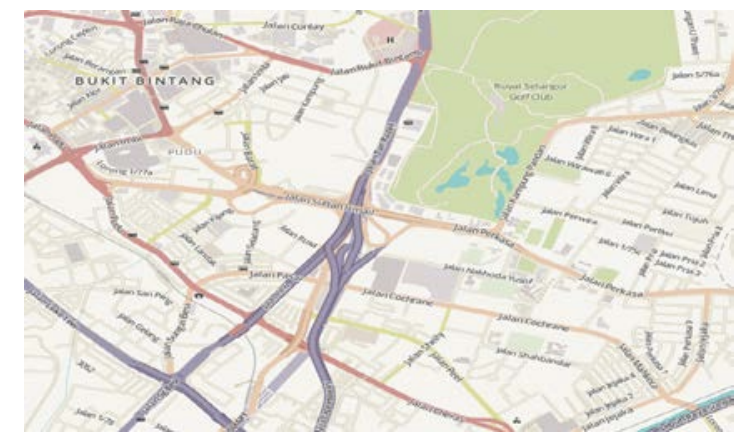

a)

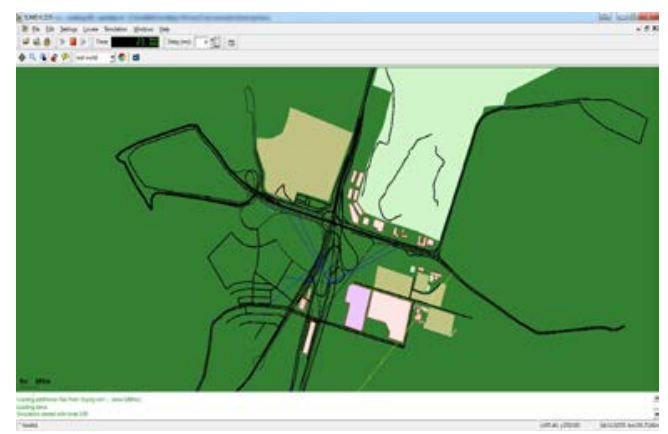

b)

Fig. 5. a) Simulation map (Jalan Kampung Pandan, Kuala Lumpur) b) Simulation scenario in SUMO

The mobility pattern generated on the map of Jalan Kampung Pandan, Kuala Lumpur (Malaysia) highway scenario, is shown in Fig. 5. The overall distance for the simulation is about $10 \mathrm{~km}$. The map consists of bidirectional lanes, varying between two and four. Vehicles are introduced into the simulation randomly, at an equal rate of five vehicles per second. The total simulation duration is $10 \mathrm{~min}$. The clustering formation process is initiated at the $30^{\text {th }}$ second, with an inter-vehicle distance of $2.5 \mathrm{~m}$ and a velocity of $30 \mathrm{~m} / \mathrm{s}$. The simulation assumed that a QCIF sequence of pre-recorded traffic used approximately 600 QCIF frames, and that multimedia content was transmitted over a three-hop path. The size of the frame was $176 \times 144$ pixels at $30 \mathrm{f} / \mathrm{s}$ for each description. 
Table 3. Simulation Parameters of LTE

\begin{tabular}{|l|l|}
\hline Parameter & Value \\
\hline eNodeB Scheduler Type & RrFfMacScheduler \\
\hline eNodeB Coverage & $7 \mathrm{~km}$ \\
\hline
\end{tabular}

Table 4. Simulation Parameters of VANET

\begin{tabular}{|l|l|}
\hline Parameter & Value \\
\hline Simulation scenario & Highway \\
\hline Simulation time & $600 \mathrm{~s}$ \\
\hline Road Length & $10 \mathrm{~km}$ \\
\hline Number of vehicles & 600 \\
\hline Maximum velocity of vehicles & $30 \mathrm{~m} / \mathrm{s}$ \\
\hline Minimum inter-vehicle distance & $2.5 \mathrm{~m}$ \\
\hline Maximum transmission range & $300 \mathrm{~m}$ \\
\hline MAX_HOP & $1,2,3$ \\
\hline MAC Layer & DCF CSMA/CA \\
\hline MAXMEMBER_CH & 5 \\
\hline HELLO_PACKET period & $200 \mathrm{~ms}$ \\
\hline HELLO_PACKET size & $64 \mathrm{bytes}$ \\
\hline MULTIMEDIA_DATA period & $1 \mathrm{~s}$ \\
\hline MULTIMEDIA_DATA size & 1024 Kbytes \\
\hline TIMER & $1 \mathrm{~s}$ \\
\hline IN_TIMER & $2 \mathrm{~s}$ \\
\hline SD_TIMER & $2 \mathrm{~s}$ \\
\hline CH_TIMER & $2 \mathrm{~s}$ \\
\hline CM_TIMER & $2 \mathrm{~s}$ \\
\hline JOIN_TIMER & $2 \mathrm{~s}$ \\
\hline COMBINE_TIMER & $2 \mathrm{~s}$ \\
\hline & \\
\hline
\end{tabular}

Tables 3 and 4 represent individual simulation parameters of the proposed LTE and VANET networks. In the proposed system, for cluster stability, the highest number of hops inside the cluster is preferred in the range of $[1,3]$. This is because as the number of hops increases, especially after the third hop in the cluster, the network becomes unstable due to an increasing number of HELLO_PACKETs broadcast in the hop, which results in higher data packet losses. The $\mathrm{CH}$ handshake process within the hops is also introduced in this research to ensure that the constructed clusters are connected.

\subsection{Simulation Results and Analysis}

By comparing it with previously proposed, mobility-based, multi-hop clustering schemes, such as VMaSC [46], DHCV [47] and ART [50], this simulation analysis evaluates the performance of our proposed mobility-based clustering scheme for multimedia content dissemination over an IEEE 802.11p-LTE hybrid VANET architecture. The reference scheme VMaSC [46] algorithm follows a relative, mobility-based cluster formation technique, where the DHCV [47] algorithm follows a mobility-related, non-overlapping cluster creation system and the ART [50] algorithm creates cluster-based, relative vehicular speed. To analyse the performance of the proposed IEEE 802.11p-LTE hybrid VANET architecture, pure VANET cluster-based data forwarding techniques are compared, along with VMaSC-LTE, DHCV-LTE and ART-LTE, where the cellular communication technology LTE integrates with the referenced VANET clustering algorithms VMaSC [46], DHCV [47] and ART [49]. The performance metrics are as follows: 
1) Average Cluster Head Duration:

This is defined as the average time period during which a $\mathrm{CH}$ remains in a leadership role until it moves to another status (e.g. SD). The average $\mathrm{CH}$ durations under different velocities within the proposed algorithm and reference schemes are presented in Fig. 6.

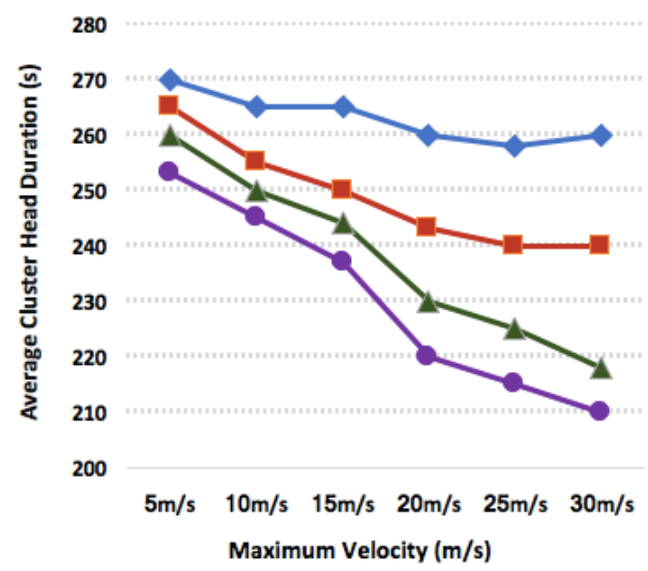

a)

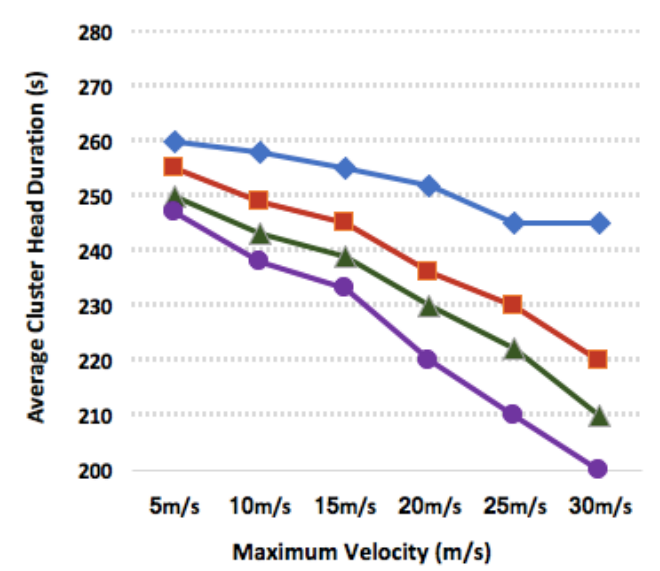

b)

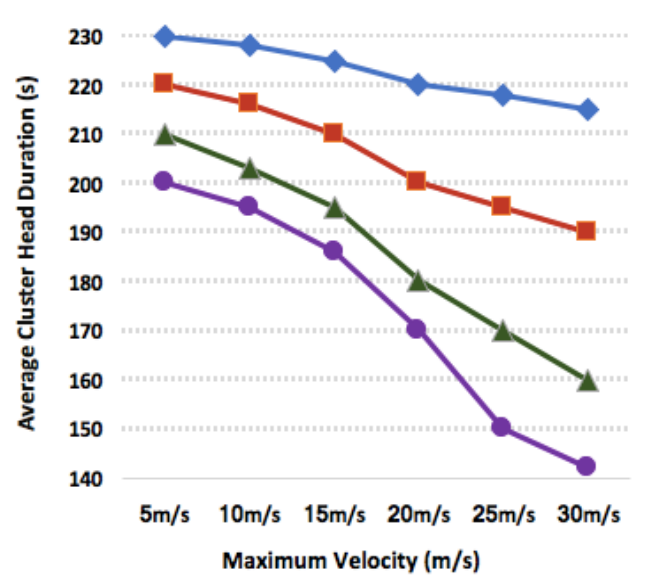

c)
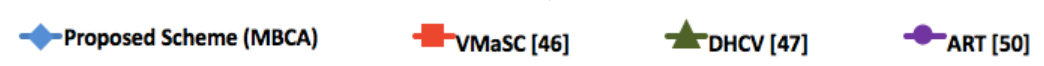

Fig. 6. Average CH duration (s), for Transmission Range (a) 100, (b) 200 and (c) $300 \mathrm{~m}$

The above graphs demonstrate that the average $\mathrm{CH}$ duration in the proposed scheme improved by about 20\%, compared to the referenced schemes at different transmission ranges. They also showed that the referenced clustering schemes tend to decrease rapidly, along with higher vehicle velocity and transmission range, whereas the proposed scheme is more stable. This is because when a vehicle's speed increases, the network topology changes rapidly. In Graphs a), b) and c), the referenced scheme VMaSC [46] gives overall a 10\% higher $\mathrm{CH}$ duration compared to DHCV [47] and ART [50]. The proposed ART [50] scheme decreases the $\mathrm{CH}$ duration very rapidly at transmission ranges of $100 \mathrm{~m}, 200 \mathrm{~m}$ and $300 \mathrm{~m}$ throughout when the velocity is increased from 15 to $30 \mathrm{~m} / \mathrm{s}$. 
Because of the presence of an SE_CH, in the proposed scheme, the metric that notably improves the $\mathrm{CH}$ duration is MAX_HOP. This can be explained in a multi-hop scenario, where a $\mathrm{CH}$ has a greater chance to stay connected and to serve CMs in its hop, whereas an SE_CH keeps the cluster's connectivity stable when the $\mathrm{CH}$ leaves the network. The $\mathrm{CH}$ handshake stabilizes cluster performance, which in turn makes $\mathrm{CHs}$ remain in $\mathrm{CH}$ status longer. Another reason for longer $\mathrm{CH}$ duration is the limitation of its cluster size. Hence, the $\mathrm{CH}$ has a greater chance to search for neighbouring vehicles to attend to as the number of hops increases.

2) Average Cluster Member Duration:

Average Cluster Member Duration is defined as the average time a vehicle stays continuously in a cluster. In the proposed algorithm, CM duration is calculated as the interval duration of an existing connected member of a cluster before the cluster moves to SD status.

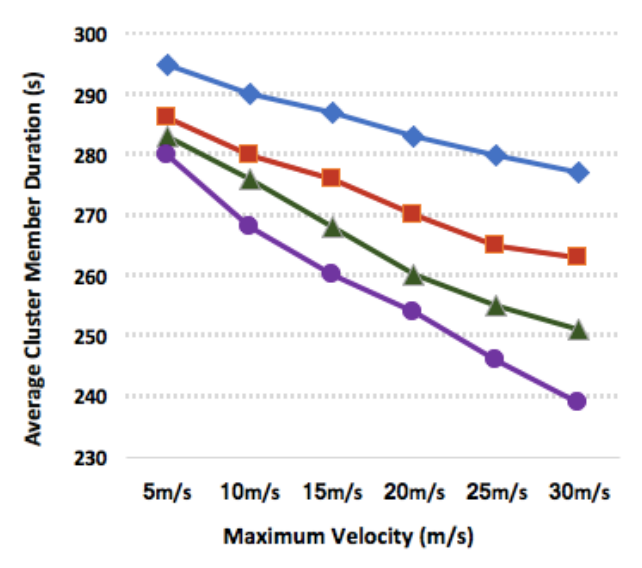

a)

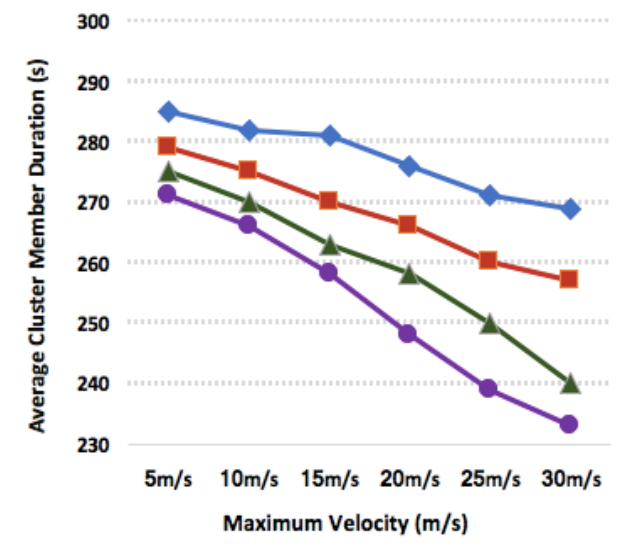

b)

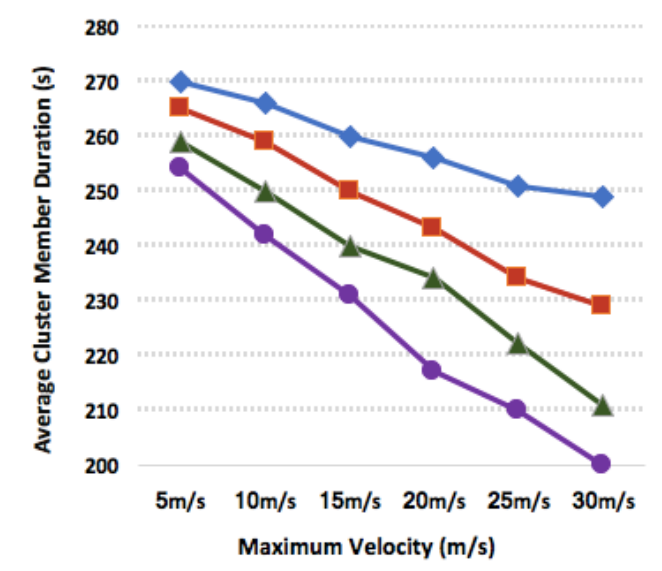

c)

-Proposed Scheme (MBCA)

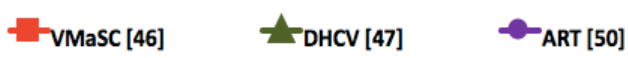

Fig. 7. Average CM Duration (s), for Transmission Range (a) 100, (b) 200 and (c) $300 \mathrm{~m}$

Fig. 7 presents the comparative average CM duration results for the proposed algorithm along with reference schemes at different velocities. The result indicates that as a vehicle's 
velocity increases, frequent topology changes shorten the CM durations considerably for all reference clustering schemes, where the proposed clustering schemes perform a $20 \%$ higher $\mathrm{CM}$ duration from the referenced schemes. This is because in a high dynamic scenario, vehicles do not receive $\mathrm{CH}$ or $\mathrm{CM}$ packets simultaneously as they travel, which results in vehicles switching to SD status or declaring themselves as SELF_CH. After a while, vehicles may receive feedback from another $\mathrm{CH}$ or be unable to discover any vehicle to connect to as CM. Eventually, vehicles' status changes decrease the CM duration for all reference schemes. In the proposed scheme, because a secondary cluster leader enables CMs to stay connected even when the $\mathrm{CH}$ drops the connection, CM duration increases in the network. The handshake process with the cluster also improves CM duration in the proposed scheme.

3) Cluster Overhead:

Cluster Overhead measures the percentage of total number of clustering-related packets generated within the VANET. The performance of the proposed clustering algorithm and reference clustering algorithms, in terms of clustering overhead at different velocities, is illustrated in Fig. 8. The results show that due to the constructed cluster structure stability in terms of $\mathrm{CH}$ and $\mathrm{CM}$ durations, the proposed MBCA overhead is smaller than that of the referenced schemes VMaSC [46], DHCV [47] and ART [50].

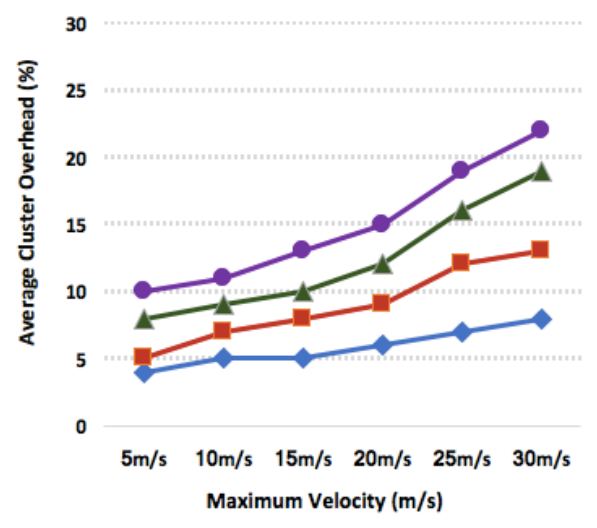

a)

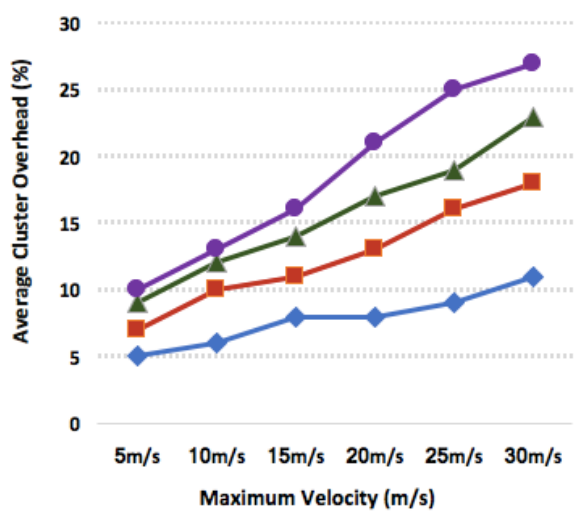

b)

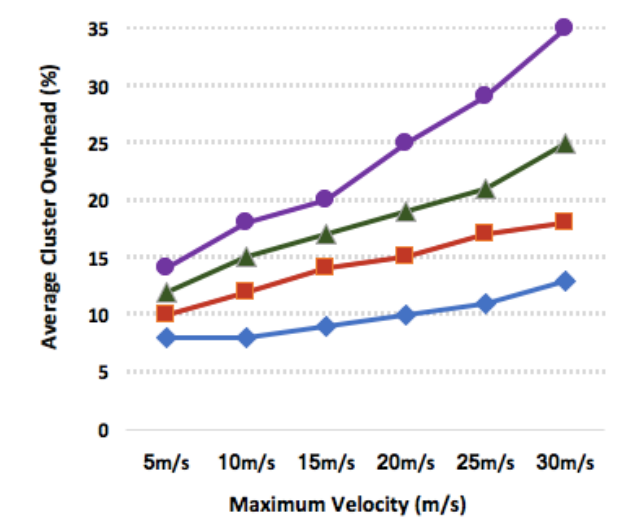

c)

\footnotetext{
-Proposed Scheme (MBCA)
}

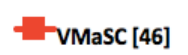

Fig. 8. Average Cluster overhead (\%), for Transmission Range (a) 100, (b) 200 and (c) $300 \mathrm{~m}$ 
A fast network topology change causes a drastic increase in clustering-related information exchange in the referenced schemes VMaSC [46], DHCV [47] and ART [50]. One of the key metrics that affects clustering overhead is the dissemination of HELLO_PACKET in multi-hop scenarios. As the number of hops increases, so does the overhead. This is because to reach the n-hop vehicles, HELLO_PACKETs are rebroadcasted by controlling the hop limit. Due to timer-based cluster maintenance in the proposed scheme, clustering is applied when necessary, which decreases the clustering overhead.

4) Data Packet Delivery Ratio (DPDR):

Fig. 9 shows the average DPDR at maximum velocities for the proposed scheme MBCA, along with the Standard IEEE 802.11p VANET, VMaSC-LTE, DHCV-LTE and ART-LTE. The results show that the DPDR of the proposed scheme MBCA shows improved performance over all other algorithms.

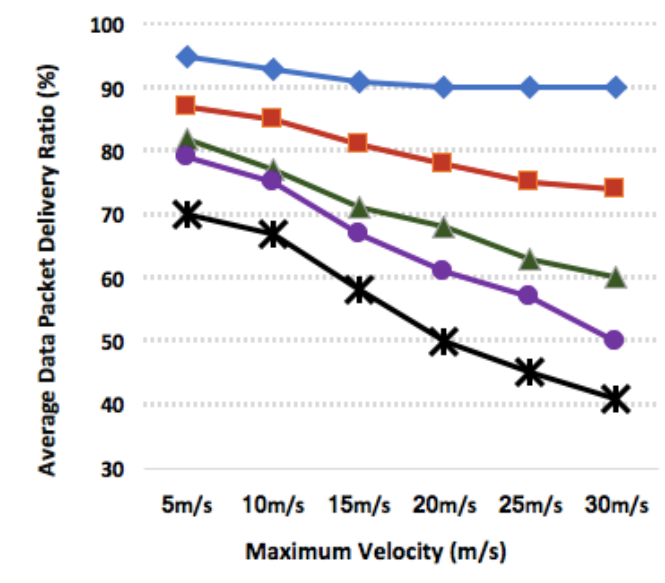

a)

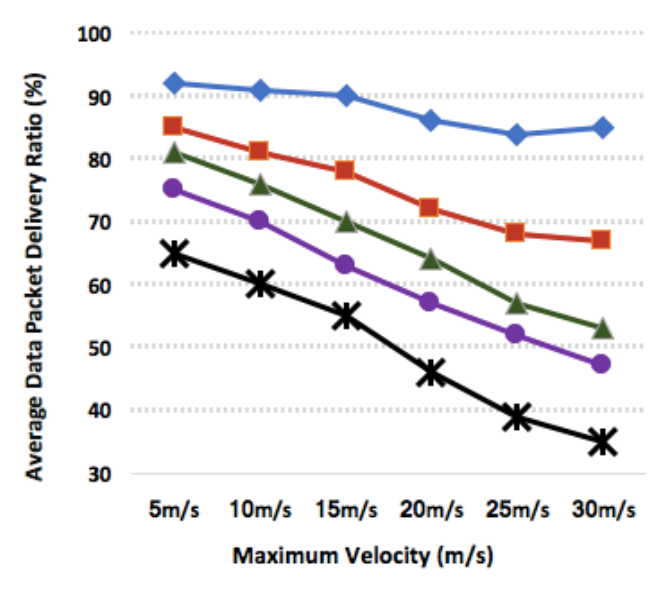

b)

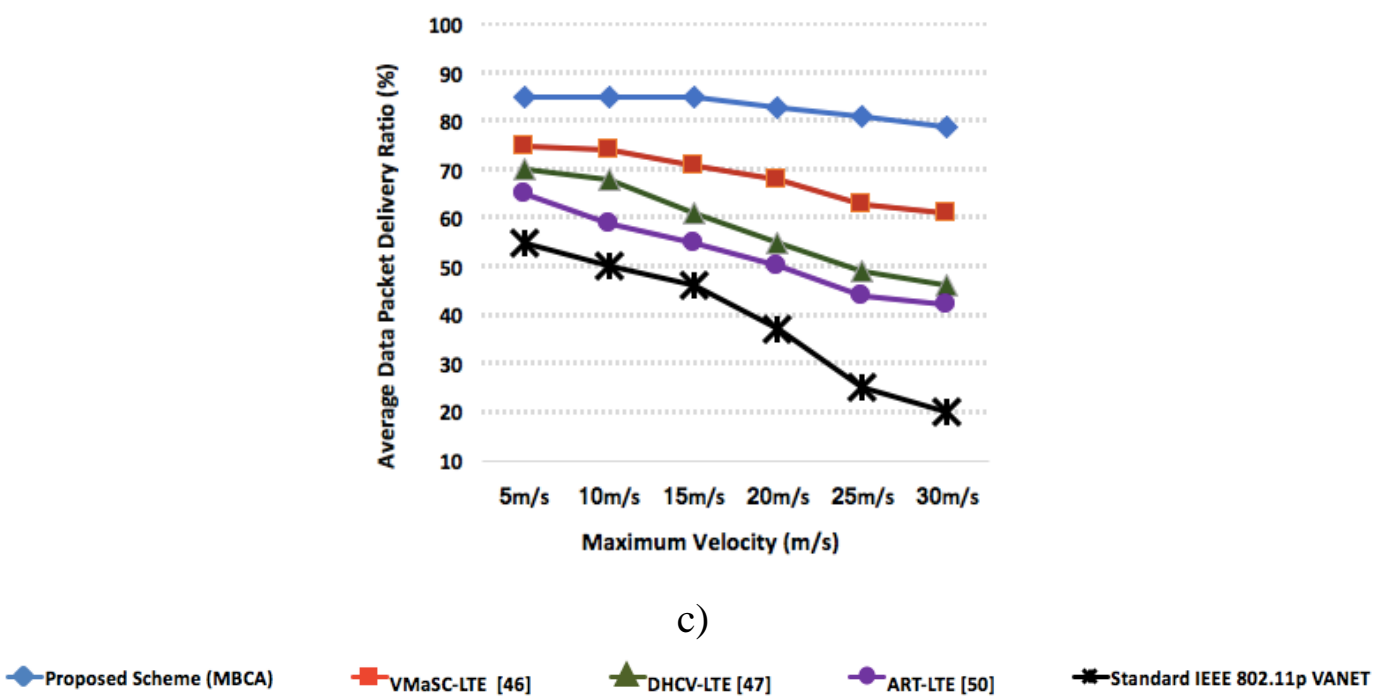

Fig. 9. Average Data Packet Delivery Ratio (\%), for transmission range

(a) 100, (b) 200 and (c) $300 \mathrm{~m}$ 
Clustering stability improvement, clustering overhead control, handshakes with the cluster and clustering overlap minimization enable the proposed clustering scheme to achieve superior DPDR performance. A high connection stability between CMs and CHs results in better clustering stability and reduces the number of status changes in the cluster. In the proposed scheme, an SE_CH enables stable connectivity within the cluster, where the reference schemes show higher connection loss in a dynamic scenario. Minimizing the clustering overhead and clustering overlap increases the success rate of data dissemination over the network. The results also show that the enhanced DPDR performance in data forwarding in the proposed scheme performs better than the referenced schemes such as the standard IEEE 802.11p VANET. The proposed IEEE 802.11p-LTE hybrid VANET architecture reduces DSRC communication by using LTE-based inter-cluster communication, which reduces the medium access conflict over the network.

5) Network Delay:

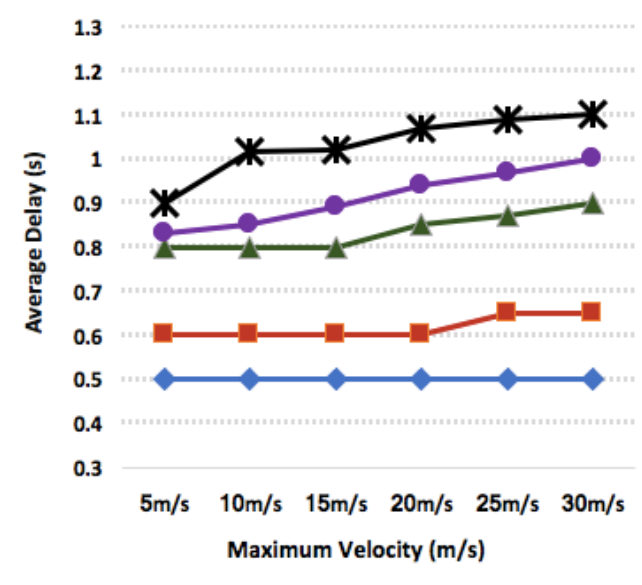

a)

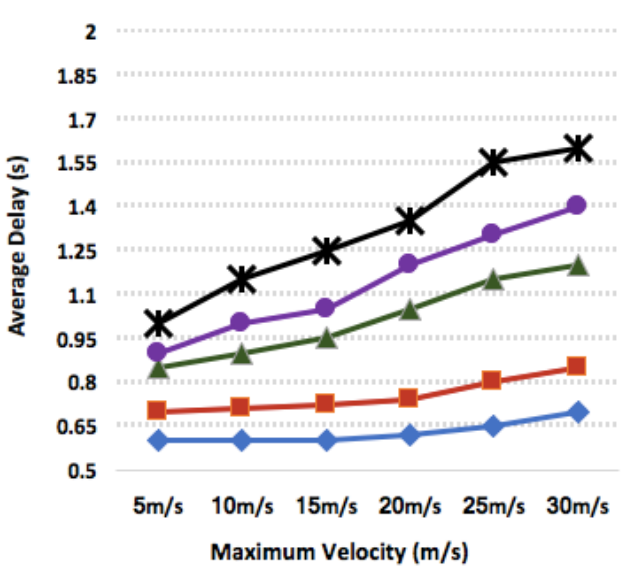

b)

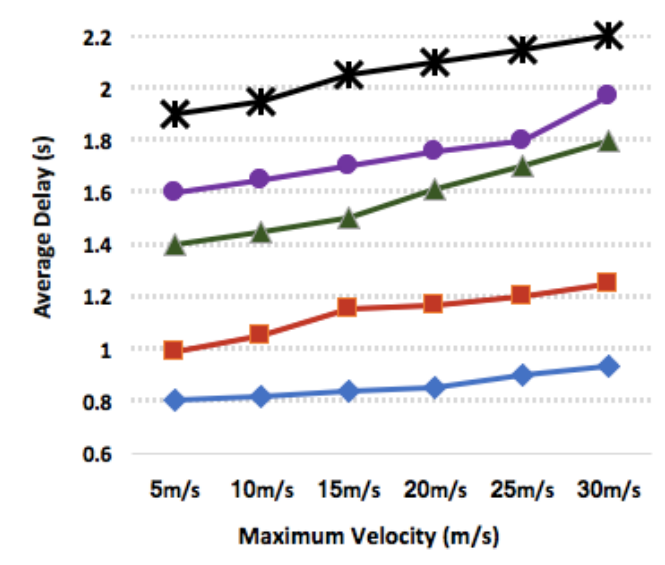

c)

$\sim$-Proposed Scheme (MBCA)

$$
\text { -VMaSC-LTE [46] }
$$

DohCV-Lte [47]

-ART-LTE [50]

$\rightarrow$-Standard IEEE 802.11p VANET

Fig. 10. Average Delay (s), for Transmission Range (a) 100, (b) 200 and (c) $300 \mathrm{~m}$ 
Fig. 10 shows the average delay trade-off at maximum velocities for the proposed clustering algorithm, along with that for standard IEEE 802.11p VANET and referenced schemes. The results show that the proposed IEEE 802.11p-LTE-based hybrid VANET architecture achieves lower delay than the referenced schemes. High clustering stability and low overhead in the network are the main reasons for low average delay in the proposed scheme. Another reason for lower network delay in the proposed scheme MBCA is the data bandwidth optimization achieved using the cellular communication technology LTE as the secondary communication link. This enables vehicles to disseminate high-bandwidth data over an LTE network rather than DSRC, which reduces data congestion flow over the network and network delay.

\section{Conclusion}

As indicated by the element versatility of VANET, it is possible for vehicles to connect to each other by establishing a strong backbone for critical road safety information by broadcasting over a network. The dynamic characteristics of VANET create major integration issues for multimedia content dissemination in a vehicular network environment. A clustering algorithm constructs an organized network pattern in which vehicles form groups to manage data dissemination in an efficient way. In this paper, we propose a mobility-based clustering algorithm to support efficient multimedia broadcasts in a vehicular network environment. The essence of our clustering approach is to use mobility metrics for cluster formation, to choose a $\mathrm{CH}$ with a similar average relative speed and a handshake process for the existing cluster, which ensures stability. The extensive simulation results show that our proposed clustering scheme enhances vehicular network performance for a multimedia broadcasting service by improving cluster stability, minimizing cluster overhead, achieving a high data packet delivery ratio and reducing network delays. To further improve our cluster scheme, our future work will focus on investigating network performance in a complex urban scenario.

\section{References}

[1] J. Gubbi, R. Buyya, S. Marusic, and M. Palaniswami, "Internet of Things (IoT): A vision, architectural elements, and future directions," Future Gener. Comput. Syst., vol. 29, pp. 1645-1660, 2013. Article (CrossRef Link)

[2] S. K. Bhoi and P. M. Khilar, "Vehicular communication: a survey," IET Networks, vol. 3, pp. 204-217, 2014. Article (CrossRef Link)

[3] G. Karagiannis, O. Altintas, E. Ekici, G. Heijenk, B. Jarupan, K. Lin, et al., "Vehicular networking: A survey and tutorial on requirements, architectures, challenges, standards and solutions," IEEE Communications Surveys \& Tutorials, 2011. Article (CrossRef Link)

[4] M. Conti and S. Giordano, "Mobile ad hoc networking: milestones, challenges, and new research directions," IEEE Communications Magazine, vol. 52, pp. 85-96, 2014. Article (CrossRef Link)

[5] M. Garai, S. Rekhis, and N. Boudriga, "Communication as a service for cloud VANETs," in Proc. of 2015 IEEE Symposium on Computers and Communication (ISCC), pp. 371-377, 2015. Article (CrossRef Link)

[6] A. Dua, N. Kumar, and S. Bawa, "A systematic review on routing protocols for Vehicular Ad Hoc Networks," Vehicular Communications, vol. 1, pp. 33-52, 2014. Article (CrossRef Link)

[7] M. A. Qureshi and R. M. Noor, "Towards Improving Vehicular Communication in Modern Vehicular Environment," in Proc. of 2013 11th International Conference on Frontiers of Information Technology, pp. 177-182, 2013. Article (CrossRef Link) 
[8] "IEEE Standard for Information technology-- Local and metropolitan area networks-- Specific requirements-- Part 11: Wireless LAN Medium Access Control (MAC) and Physical Layer (PHY) Specifications Amendment 6: Wireless Access in Vehicular Environments," IEEE Std 802.11p-2010 (Amendment to IEEE Std 802.11-2007 as amended by IEEE Std 802.11k-2008, IEEE Std 802.11r-2008, IEEE Std 802.11y-2008, IEEE Std 802.11n-2009, and IEEE Std 802.11w-2009), pp. 1-51, 2010. Article (CrossRef Link)

[9] J. P. Singh and R. S. Bali, "A Hybrid Backbone Based Clustering Algorithm for Vehicular Ad-Hoc Networks," Procedia Computer Science, vol. 46, pp. 1005-1013, 2015. Article (CrossRef Link)

[10] N. Wisitpongphan, F. Bai, P. Mudalige, V. Sadekar, and O. Tonguz, "Routing in Sparse Vehicular Ad Hoc Wireless Networks," IEEE Journal on Selected Areas in Communications, vol. 25, pp. 1538-1556, 2007. Article (CrossRef Link)

[11] M. Kakkasageri and S. Manvi, "Connectivity and mobility aware dynamic clustering in VANETs," International Journal of Future Computer and Communication, vol. 3, p. 5, 2014. Article (CrossRef Link)

[12] M. Dixit, R. Kumar, and A. K. Sagar, "VANET: Architectures, research issues, routing protocols, and its applications," in 2016 International Conference on Computing, Communication and Automation (ICCCA), 2016, pp. 555-561. Article (CrossRef Link)

[13] N. Wisitpongphan, O. K. Tonguz, J. S. Parikh, P. Mudalige, F. Bai, and V. Sadekar, "Broadcast storm mitigation techniques in vehicular ad hoc networks," IEEE Wireless Communications, vol. 14, pp. 84-94, 2007. Article (CrossRef Link)

[14] O. Tonguz, N. Wisitpongphan, F. Bait, P. Mudaliget, and V. Sadekart, "Broadcasting in VANET," in 2007 mobile networking for vehicular environments, pp. 7-12, 2007. Article (CrossRef Link)

[15] S. Vodopivec, J. Bešter, and A. Kos, "A survey on clustering algorithms for vehicular ad-hoc networks," in Proc. of 2012 35th International Conference on Telecommunications and Signal Processing (TSP), pp. 52-56, 2012. Article (CrossRef Link)

[16] G. Araniti, C. Campolo, M. Condoluci, A. Iera, and A. Molinaro, "LTE for vehicular networking: a survey," IEEE Communications Magazine, vol. 51, pp. 148-157, 2013. Article (CrossRef Link)

[17] G. Remy, S. M. Senouci, F. Jan, and Y. Gourhant, "LTE4V2X: LTE for a Centralized VANET Organization," in Proc. of 2011 IEEE Global Telecommunications Conference - GLOBECOM 2011, pp. 1-6, 2011. Article (CrossRef Link)

[18] D. Krajzewicz, M. Hartinger, G. Hertkorn, P. Mieth, C. Rössel, J. Zimmer, et al., "Using the Road Traffic Simulation "SUMO" for Educational Purposes," Traffic and Granular Flow '03, S. P. Hoogendoorn, S. Luding, P. H. L. Bovy, M. Schreckenberg, and D. E. Wolf, Eds., ed Berlin, Heidelberg: Springer Berlin Heidelberg, pp. 217-22, 2005. Article (CrossRef Link)

[19] A. Varga, "OMNeT++," in Modeling and Tools for Network Simulation, K. Wehrle, M. Güneş, and J. Gross, Eds., ed Berlin, Heidelberg: Springer Berlin Heidelberg, pp. 35-59, 2010. Article (CrossRef Link)

[20] C. Sommer, J. Härri, F. Hrizi, B. Schünemann, and F. Dressler, "Simulation Tools and Techniques for Vehicular Communications and Applications," Vehicular ad hoc Networks: Standards, Solutions, and Research, C. Campolo, A. Molinaro, and R. Scopigno, Eds., ed Cham: Springer International Publishing, pp. 365-392, 2015. Article (CrossRef Link)

[21] C. Cooper, D. Franklin, M. Ros, F. Safaei, and M. Abolhasan, "A Comparative Survey of VANET Clustering Techniques," IEEE Communications Surveys \& Tutorials, vol. 19, pp. 657-681, 2017. Article (CrossRef Link)

[22] R. Agarwal and D. Motwani, "Survey of clustering algorithms for MANET," arXiv preprint arXiv:0912.2303, 2009. Article (CrossRef Link)

[23] P. Basu, N. Khan, and T. D. Little, "A mobility based metric for clustering in mobile ad hoc networks," in Dis Proc. of tributed computing systems workshop, 2001 international conference on, pp. 413-418, 2001. Article (CrossRef Link)

[24] A. Bentaleb, A. Boubetra, and S. Harous, "Survey of clustering schemes in mobile ad hoc networks," Communications and Network, vol. 5, p. 8, 2013. Article (CrossRef Link) 
[25] C. R. Lin and M. Gerla, "Adaptive clustering for mobile wireless networks," IEEE Journal on Selected Areas in Communications, vol. 15, pp. 1265-1275, 1997. Article (CrossRef Link)

[26] M. Ni, Z. Zhong, and D. Zhao, "MPBC: A Mobility Prediction-Based Clustering Scheme for Ad Hoc Networks," IEEE Transactions on Vehicular Technology, vol. 60, pp. 4549-4559, 2011. Article (CrossRef Link)

[27] R. P. Selvam and V. Palanisamy, "Stable and flexible weight based clustering algorithm in mobile ad hoc networks," International Journal of Computer Science and Information Technologies, vol. 2, pp. 824-828, 2011. Article (CrossRef Link)

[28] Y. Xu, S. Bien, Y. Mori, J. Heidemann, and D. Estrin, "Topology control protocols to conserve energy in wireless ad hoc networks," Center for Embedded Network Sensing, 2003. Article (CrossRef Link)

[29] J. Y. Yu and P. H. J. Chong, "3hBAC (3-hop between adjacent clusterheads): a novel non-overlapping clustering algorithm for mobile ad hoc networks," in Proc. of 2003 IEEE Pacific Rim Conference on Communications Computers and Signal Processing (PACRIM 2003) (Cat. No.03CH37490), vol.1, pp. 318-321, 2013. Article (CrossRef Link)

[30] A. Ephremides, J. E. Wieselthier, and D. J. Baker, "A design concept for reliable mobile radio networks with frequency hopping signaling," Proceedings of the IEEE, vol. 75, pp. 56-73, 1987. Article (CrossRef Link)

[31] M. Gerla and J. Tzu-Chieh Tsai, "Multicluster, mobile, multimedia radio network," Wireless Networks, vol. 1, pp. 255-265, 1995. Article (CrossRef Link)

[32] Y. Zhang and J. M. Ng, "A Distributed Group Mobility Adaptive Clustering Algorithm for Mobile Ad Hoc Networks," in Proc. of 2008 IEEE International Conference on Communications, pp. 3161-3165, 2008. Article (CrossRef Link)

[33] C. Konstantopoulos, D. Gavalas, and G. Pantziou, "Clustering in mobile ad hoc networks through neighborhood stability-based mobility prediction," Computer Networks, vol. 52, pp. 1797-1824, 2008. Article (CrossRef Link)

[34] C. Shea, B. Hassanabadi, and S. Valaee, "Mobility-Based Clustering in VANETs Using Affinity Propagation," in Proc. of GLOBECOM 2009 - 2009 IEEE Global Telecommunications Conference, pp. 1-6, 2009. Article (CrossRef Link)

[35] N. Maslekar, M. Boussedjra, J. Mouzna, and L. Houda, "Direction based clustering algorithm for data dissemination in vehicular networks," in Proc. of 2009 IEEE Vehicular Networking Conference (VNC), pp. 1-6, 2009. Article (CrossRef Link)

[36] R. T. Goonewardene, F. H. Ali, and E. Stipidis, "Robust mobility adaptive clustering scheme with support for geographic routing for vehicular ad hoc networks," IET Intelligent Transport Systems, vol. 3, pp. 148-158, 2009. Article (CrossRef Link)

[37] Z. Y. Rawashdeh and S. M. Mahmud, "A novel algorithm to form stable clusters in vehicular ad hoc networks on highways," EURASIP Journal on Wireless Communications and Networking, vol. 2012, p. 15, 2012. Article (CrossRef Link)

[38] Z. Y. Rawshdeh and S. M. Mahmud, "Toward Strongley Connected Clustering Structure in Vehicular Ad Hoc Networks," in Proc. of 2009 IEEE 70th Vehicular Technology Conference Fall, pp. 1-5, 2009. Article (CrossRef Link)

[39] E. Souza, I. Nikolaidis, and P. Gburzynski, "A New Aggregate Local Mobility; Clustering Algorithm for VANETs," in Proc. of 2010 IEEE International Conference on Communications, pp. 1-5, 2010. Article (CrossRef Link)

[40] S. Vodopivec, J. Bešter, and A. Kos, "A multihoming clustering algorithm for vehicular ad hoc networks," International Journal of Distributed Sensor Networks, 2014. Article (CrossRef Link)

[41] G. Wolny, "Modified DMAC Clustering Algorithm for VANETs," in Proc. of 2008 Third International Conference on Systems and Networks Communications, pp. 268-273, 2008. Article (CrossRef Link)

[42] K. A. Hafeez, L. Zhao, Z. Liao, and B. N. W. Ma, "A fuzzy-logic-based cluster head selection algorithm in VANETs," in Proc. of 2012 IEEE International Conference on Communications (ICC), pp. 203-207, 2012. Article (CrossRef Link) 
[43] Z. Zhang, A. Boukerche, and R. Pazzi, "A novel multi-hop clustering scheme for vehicular ad-hoc networks," in Proc. of the 9th ACM international symposium on Mobility management and wireless access, pp. 19-26, 2011. Article (CrossRef Link)

[44] Y. Chen, M. Fang, S. Shi, W. Guo, and X. Zheng, "Distributed multi-hop clustering algorithm for VANETs based on neighborhood follow," EURASIP Journal on Wireless Communications and Networking, vol. 2015, pp. 1-12, 2015. Article (CrossRef Link)

[45] S. Ucar, S. C. Ergen, and O. Ozkasap, "VMaSC: Vehicular multi-hop algorithm for stable clustering in Vehicular Ad Hoc Networks," in Proc. of 2013 IEEE Wireless Communications and Networking Conference (WCNC), pp. 2381-2386, 2013. Article (CrossRef Link)

[46] S. Ucar, S. C. Ergen, and O. Ozkasap, "Multihop-Cluster-Based IEEE 802.11p and LTE Hybrid Architecture for VANET Safety Message Dissemination," IEEE Transactions on Vehicular Technology, vol. 65, pp. 2621-2636, 2016. Article (CrossRef Link)

[47] M. Azizian, S. Cherkaoui, and A. S. Hafid, "A distributed D-hop cluster formation for VANET," in Proc. of 2016 IEEE Wireless Communications and Networking Conference, pp. 1-6, 2016. Article (CrossRef Link)

[48] M. Azizian, S. Cherkaoui, and A. S. Hafid, "DCEV: A distributed cluster formation for VANET based on end-to-end realtive mobility," in Proc. of 2016 International Wireless Communications and Mobile Computing Conference (IWCMC), pp. 287-291, 2016. Article (CrossRef Link)

[49] M. Azizian, S. Cherkaoui, and A. Hafid, "An Optimized Flow Allocation in Vehicular Cloud," IEEE Access, vol. 4, pp. 6766-6779, 2016. Article (CrossRef Link)

[50] Z. Merhi, O. Tahan, S. Abdul-Nabi, A. Haj-Ali, and M. Bayoumi, "Decentralized clustering in VANET using adaptive resonance theory," in Proc. of 2015 IEEE International Conference on Electronics, Circuits, and Systems (ICECS), pp. 205-208, 2015. Article (CrossRef Link)

[51] P. Hubballi, A. V. Sutagundar, and R. Belagali, "Agent based dynamic clustering for hybrid VANET (ADCHV)," in Proc. of 2016 IEEE International Conference on Recent Trends in Electronics, Information \& Communication Technology (RTEICT), pp. 382-386, 2016. Article (CrossRef Link)

[52] M. S. Aman, H. Jiang, C. Quint, K. Yelamarthi, and A. Abdelgawad, "Reliability evaluation of iBeacon for micro-localization," in Proc. of 2016 IEEE 7th Annual Ubiquitous Computing, Electronics \& Mobile Communication Conference (UEMCON), pp. 1-5, 2016. Article (CrossRef Link)

[53] S. Sheikholeslam and C. A. Desoer, "Longitudinal control of a platoon of vehicles," in Proc. of American Control Conference, 1990, pp. 291-296, 1990. Article (CrossRef Link)

[54] S. S. Yau and W. Gao, "Multi-hop Clustering Based on Neighborhood Benchmark in Mobile Ad-hoc Networks," Mobile Networks and Applications, vol. 12, pp. 381-391, 2007. Article (CrossRef Link) 

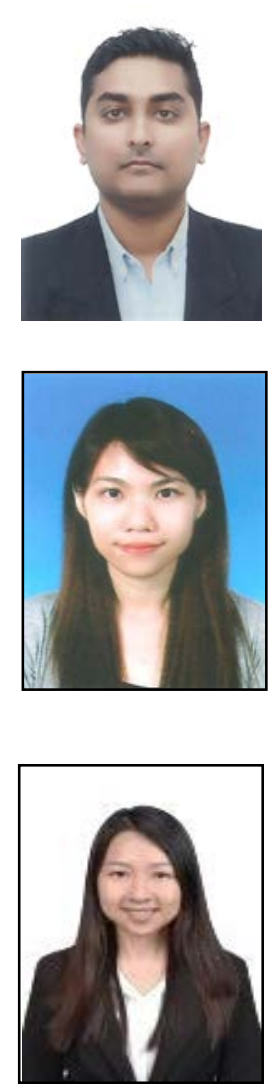

Mohammad Syfullah is a MSc in Engineering by Research candidate at SEGi University, Selangor, Malaysia. He received his BEng (with honours) degree in Electrical and Electronics Engineering from Glyndwr University, Wrexham, UK, in 2012. His research interests include wireless communications and networking, intelligent transportation systems, Vehicular Ad-hoc Network (VANET), vehicle-to-everything communication, clustering algorithms, MAC protocols, routing protocols, VANET applications, and network security.

Joanne Mun-Yee Lim received her Bachelor of Engineering (with honours) from Monash University in 2008, her Master of Engineering Degree from University Malaya in 2011, and her PhD (Engineering) from Multimedia University in 2016. She is also a member of The Institution of Engineers Malaysia and a professional engineer of the Board of Engineers Malaysia. She joined the Department of Electrical Engineering in Asia Pacific University as a Lecturer in 2011. She is currently a Lecturer in the Department of Electrical and Computer Systems Engineering, School of Engineering, Monash University Malaysia. Her research interest includes Vehicular Ad-hoc Network (VANET), mobile IPv6 based networks, artificial intelligence, control systems, optimization schemes, robotic design and applications.

Siaw Fei Lu graduated from Universiti Tenaga Nasional, Malaysia in 2003 with a Bachelor's degree in Electrical and Electronics Engineering. After working in the industry, she pursued her Masters in Engineering and Science as well as PhD in Engineering from 2007 to 2014 at University Tunku Abdul Rahman, Malaysia. At the university, Dr Siaw researched on the concentrator solar photovoltaic module, characterization of III-V triple-junction solar cells, and assisted in obtaining research fund from MESITA. In 2015 she joined the Department of Electrical and Electronics Engineering at SEGi University as a Senior Lecturer. Her research work focuses on the optimization of solar photovoltaic systems. This work would contribute in efforts of reducing the dependence on fossil fuels and decreasing greenhouse gas emissions. 